\title{
General exponential dichotomies on time scales and parameter dependence of roughness
}

\author{
Jimin Zhang, Yajun Song and Zhitao Zhao*
}

\section{"Correspondence:}

zhitaozhao0808@126.com

School of Mathematical Sciences,

Heilongjiang University, 74 Xuefu

Street, Harbin, Heilongjiang 150080,

P.R. China

\begin{abstract}
This paper focuses on a new notion called the general exponential dichotomy on time scales, which is more general and contains as special cases most versions of dichotomies on the continuous systems and discrete systems. We establish the existence of parameter dependence of roughness for the general exponential dichotomy on time scales under sufficiently small linear perturbation. Moreover, we also show that the stable and unstable subspaces of general exponential dichotomies for the perturbed system are Lipschitz continuous for the parameters.
\end{abstract}

MSC: 34N05; 34D09

Keywords: time scales; general exponential dichotomy; roughness

\section{Introduction}

The notion of exponential dichotomies extends the idea of hyperbolicity from autonomous systems to nonautonomous systems and gives a direct sum of the stable and unstable subspaces for the splitting of the state space $[1,2]$. The exponential dichotomy together with its variants and extensions has been widely studied and discussed and plays a central role in the study of the nonautonomous systems [3-15]. In particular, the roughness of dichotomies states that the behavior of a dichotomy does not change much under sufficiently small linear perturbations and has been extensively studied for the continuous systems $[1,5,6,9,14,16-20]$ and the discrete systems [6, 21-23].

The theory of dynamic equations on time scales, which originates from [24, 25], is related not only to the set of real numbers (continuous systems) and the set of integers (discrete systems) but also to more general time scales (an arbitrary nonempty closed subset of the real numbers $\mathbb{R})[26,27]$. The concept of exponential dichotomies on time scales is a very important method and tool to explore the dynamic behavior of nonautonomous dynamic systems on time scales [28-38]. However, we note that there exist various different notions of dichotomies and different kinds of dichotomic behavior in the continuous systems and the discrete systems. It is of great interest to look for more general types of dichotomies on time scales in order to unify the notions of dichotomies in the continuous and discrete case. The main novelty of our work is that we introduce a new notion called the general exponential dichotomy on time scales, which includes and extends the existing notions of dichotomies for the continuous systems and the discrete systems usually found in the literature. Moreover, we also discuss parameter dependence of roughness for

@2013 Zhang et al.; licensee Springer. This is an Open Access article distributed under the terms of the Creative Commons Attribution License (http://creativecommons.org/licenses/by/2.0), which permits unrestricted use, distribution, and reproduction in any medium, provided the original work is properly cited. 
the general exponential dichotomy on time scales under sufficiently small linear perturbation.

The content of this paper is as follows. In Section 2, we define a new notion called the general exponential dichotomy on time scales for the linear dynamical system on time scales. Then we establish the existence of parameter dependence of roughness for the general exponential dichotomy on time scales in Section 3. Particularly, the stable and unstable subspaces of general exponential dichotomies for the perturbed system are Lipschitz continuous for the parameters.

\section{General exponential dichotomy on time scales}

In this section, we first introduce some basic knowledge and definitions on time scales, which can be found in $[24,25]$.

Let $\mathbb{T}$ be a time scale, i.e., an arbitrary nonempty closed subset of the real numbers $\mathbb{R}$. $\sigma: \mathbb{T} \rightarrow \mathbb{T}$ is the forward jump operator of $\mathbb{T}$ and $\mu(t)=\sigma(t)-t$ is a graininess function. Throughout this paper, the time scale $\mathbb{T}$ is assumed to be unbounded above and below. $\mathrm{C}_{\mathrm{rd}}(\mathbb{T}, \mathbb{R})$ denotes the set of rd-continuous functions $g: \mathbb{T} \rightarrow \mathbb{R} \cdot \mathcal{R}^{+}(\mathbb{T}, \mathbb{R}):=\{g \in$ $\left.C_{\mathrm{rd}}(\mathbb{T}, \mathbb{R}): 1+\mu(t) g(t)>0, t \in \mathbb{T}\right\}$ is the space of positively regressive functions.

Define

$$
\begin{aligned}
& (\varphi \oplus \psi)(t):=\varphi(t)+\psi(t)+\mu(t) \varphi(t) \psi(t), \\
& \ominus \varphi:=-\frac{\varphi(t)}{1+\mu(t) \varphi(t)}, \\
& (\omega \odot \varphi)(t):=\lim _{h \backslash \mu(t)} \frac{(1+h \varphi(t))^{\omega}-1}{h}
\end{aligned}
$$

for a given $\omega \in \mathbb{R}^{+}$and for any $t \in \mathbb{T}, \varphi, \psi \in \mathcal{R}^{+}(\mathbb{T}, \mathbb{R})$. For any $\varphi \in \mathcal{R}^{+}(\mathbb{T}, \mathbb{R})$, define the exponential function by

$$
e_{\varphi}(t, s)=\exp \left\{\int_{s}^{t} \zeta_{\mu(\tau)}(\varphi(\tau)) \Delta \tau\right\} \quad \text { with } \zeta_{h}(z)= \begin{cases}z & \text { if } h=0 \\ \log (1+h z) / h & \text { if } h \neq 0\end{cases}
$$

for $s, t \in \mathbb{T}$, where Log is the principal logarithm.

Let

$$
[\varphi]^{*}:=\sup _{t \in \mathbb{T}}(\varphi(t)), \quad[\varphi]_{*}:=\inf _{t \in \mathbb{T}}(\varphi(t))
$$

for any bounded function $\varphi \in \mathrm{C}_{\mathrm{rd}}(\mathbb{T}, \mathbb{R})$ and define

$$
\kappa_{1}:=\min \left\{t \in \mathbb{T} \cap \mathbb{R}^{+}\right\}, \quad \kappa_{2}:=\max \left\{t \in \mathbb{T} \cap \mathbb{R}^{-}\right\} .
$$

Then we have

$$
\begin{aligned}
& \lim _{t \rightarrow \infty} e_{\ominus \varphi}(t, \tau)=0, \quad \lim _{\tau \rightarrow-\infty} e_{\ominus \varphi}(t, \tau)=0 \\
& e_{\varphi}\left(t, \kappa_{1}\right) \geq 1 \quad \text { for } \kappa_{1} \leq t, \quad e_{\varphi}\left(\kappa_{2}, t\right) \geq 1 \quad \text { for } t \leq \kappa_{2},
\end{aligned}
$$

where $0<[\varphi]_{*}$. 
Let $(X,\|\cdot\|)$ be a Banach space and $\mathscr{B}(X)$ be the space of bounded linear operators defined on $X$. We consider the linear system on time scales

$$
x^{\Delta}=A(t) x,
$$

where $A \in \mathrm{C}_{\mathrm{rd}}(\mathbb{T}, \mathscr{B}(X))$. Let $T(t, s)$ be the evolution operator satisfying $T(t, s) x(s)=x(t)$ for $t, s \in \mathbb{T}$ and any solution $x(t)$ of system (2.1). Moreover, we also assume that $T(t, t)=1$ and $T(t, \tau) T(\tau, s)=T(t, s)$ for any $t, \tau, s \in \mathbb{T}$, which imply that $T(t, s)$ is invertible.

Now we introduce a new notion called the general exponential dichotomy on time scales.

Definition 2.1 System (2.1) is said to admit a general exponential dichotomy on a time scale $\mathbb{T}$ if there exist projections $P(t)$ such that

$$
P(t) T(t, s)=T(t, s) P(s), \quad t, s \in \mathbb{T},
$$

and there exist a constant $K>0, L_{1}, L_{2}: \mathbb{T} \times \mathbb{T} \rightarrow \mathbb{R}^{+}$and bounded functions $a, b \in$ $\mathrm{C}_{\mathrm{rd}}\left(\mathbb{T}, \mathbb{R}^{+}\right)$with $0<[a]_{*}, 0<[b]_{*}$ such that, for $\kappa_{1} \leq s$,

$$
\begin{array}{ll}
\|T(t, s) P(s)\| \leq K L_{1}\left(s, \kappa_{1}\right) e_{\ominus a}(t, s), & s \leq t, \\
\|T(t, s) Q(s)\| \leq K L_{2}\left(s, \kappa_{1}\right) e_{\ominus b}(s, t), \quad t \leq s
\end{array}
$$

hold and for $s \leq \kappa_{2}$,

$$
\begin{array}{ll}
\|T(t, s) P(s)\| \leq K L_{1}\left(\kappa_{2}, s\right) e_{\ominus a}(t, s), & s \leq t, \\
\|T(t, s) Q(s)\| \leq K L_{2}\left(\kappa_{2}, s\right) e_{\ominus b}(s, t), & t \leq s
\end{array}
$$

hold, where $Q(t)=\operatorname{Id}-P(t)$ is the complementary projection of $P(t)$.

In order to facilitate the reader's understanding, we now consider some specific examples of general exponential dichotomies on different time scales.

Example 2.1 Let $\mathbb{T}=\mathbb{R}$, then $\mu(t)=0, \kappa_{1}=\kappa_{2}=0$.

If functions $a, b$ are positive constants, then the general exponential dichotomy on time scales reduces to the exponential dichotomy ( $L_{1}$ and $L_{2}$ are positive constants) [1] by

$$
\|T(t, s) P(s)\| \leq K L_{1} e^{-a(t-s)}, \quad s \leq t, \quad\|T(t, s) Q(s)\| \leq K L_{2} e^{-b(s-t)}, \quad t \leq s
$$

and the nonuniform exponential dichotomy $\left(L_{1}\left(t_{1}, t_{2}\right)=L_{2}\left(t_{1}, t_{2}\right)=e^{\varepsilon\left(t_{1}-t_{2}\right)}\right.$ and $\varepsilon$ is a positive constant) [5] by

$$
\|T(t, s) P(s)\| \leq K e^{-a(t-s)+\varepsilon|s|}, \quad s \leq t, \quad\|T(t, s) Q(s)\| \leq K e^{-b(s-t)+\varepsilon|s|}, \quad t \leq s .
$$


If functions $a=b$, then we get the generalized exponential dichotomy $\left(L_{1}, L_{2}\right.$ are positive constants) $[10,11]$ by

$$
\begin{array}{ll}
\|T(t, s) P(s)\| \leq K L_{1} \exp \left(-\int_{s}^{t} a(\tau) d \tau\right), & s \leq t, \\
\|T(t, s) Q(s)\| \leq K L_{2} \exp \left(-\int_{t}^{s} a(\tau) d \tau\right), & t \leq s .
\end{array}
$$

Let $h, k: \mathbb{R}^{+} \rightarrow \mathbb{R}^{+}$, and let the functions $L_{1}, L_{2}$ be positive constants. If $a(t)=h^{\prime}(t) / h(t)$, $b(t)=k^{\prime}(t) / k(t)$, then we obtain the $(h, k)$-dichotomy [14] by

$$
\begin{array}{ll}
\|T(t, s) P(s)\| \leq K L_{1}(h(t) / h(s))^{-1}, & s \leq t, \\
\|T(t, s) Q(s)\| \leq K L_{2}(k(s) / k(t))^{-1}, & t \leq s .
\end{array}
$$

If $\mathbb{T}=\mathbb{R}^{+}$and $\eta_{i}, i=1,2,3$, are positive constants, then Definition 2.1 agrees with the nonuniform polynomial dichotomy $\left(a(t)=\eta_{1} /(t+1), b(t)=\eta_{2} /(t+1)\right.$ and $L_{1}\left(t_{1}, t_{2}\right)=$ $\left.L_{2}\left(t_{1}, t_{2}\right)=\left(t_{1}-t_{2}+1\right)^{\eta_{3}}\right)[7]$ by

$$
\begin{aligned}
& \|T(t, s) P(s)\| \leq K\left(\frac{t+1}{s+1}\right)^{-\eta_{1}}(s+1)^{\eta_{3}}, \quad s \leq t, \\
& \|T(t, s) Q(s)\| \leq K\left(\frac{s+1}{t+1}\right)^{-\eta_{2}}(s+1)^{\eta_{3}}, \quad t \leq s,
\end{aligned}
$$

the $\rho$-nonuniform exponential dichotomy $\left(a(t)=\eta_{1} \rho^{\prime}(t), b(t)=\eta_{2} \rho^{\prime}(t), L_{1}\left(t_{1}, t_{2}\right)=L_{2}\left(t_{1}\right.\right.$, $\left.\left.t_{2}\right)=e^{\eta_{3} \rho\left(t_{1}-t_{2}\right)}\right)[6]$ by

$$
\begin{aligned}
& \|T(t, s) P(s)\| \leq K e^{-\eta_{1}(\rho(t)-\rho(s))+\eta_{3} \rho(s)}, \quad s \leq t, \\
& \|T(t, s) Q(s)\| \leq K e^{-\eta_{2}(\rho(s)-\rho(t))+\eta_{3} \rho(s)}, \quad t \leq s
\end{aligned}
$$

and the nonuniform $(\mu, v)$-dichotomy $\left(a(t)=\eta_{1} \mu^{\prime}(t) / \mu(t), b(t)=\eta_{2} \mu^{\prime}(t) / \mu(t), L_{1}\left(t_{1}, t_{2}\right)=\right.$ $\left.L_{2}\left(t_{1}, t_{2}\right)=v\left(t_{1}-t_{2}\right)^{\eta_{3}}\right)[9]$ by

$$
\begin{aligned}
& \|T(t, s) P(s)\| \leq K(\mu(t) / \mu(s))^{-\eta_{1}} v(s)^{\eta_{3}}, \quad s \leq t, \\
& \|T(t, s) Q(s)\| \leq K(\mu(s) / \mu(t))^{-\eta_{2}} v(s)^{\eta_{3}}, \quad t \leq s .
\end{aligned}
$$

Example 2.2 If $\mathbb{T}=\mathbb{Z}$, then $\mu(t)=1, \kappa_{1}=\kappa_{2}=0$.

Carrying out similar arguments as those in Example 2.1, we conclude that the general exponential dichotomy on time scales includes the existing dichotomies for the linear discrete system as special cases such as the uniform exponential dichotomy [1], $(h, k)$-dichotomy [23], nonuniform exponential dichotomy [5], nonuniform polynomial dichotomy [21], $\rho$-nonuniform exponential dichotomy [6], nonuniform $(\mu, v)$-dichotomy $[8,22]$.

Example 2.3 Let $\mathbb{T}=h \mathbb{Z}, h>0$, and let the functions $a, b$ be positive constants. 
We have $\mu(t)=h, \kappa_{1}=\kappa_{2}=0$. Let $c$ be a positive constant and $L_{1}\left(t_{1}, t_{2}\right)=L_{2}\left(t_{1}, t_{2}\right)=$ $e_{c}\left(t_{1}, t_{2}\right)$. Then (2.2) and (2.3) reduce to

$$
\begin{aligned}
& \|T(t, s) P(s)\| \leq\left(\frac{1}{1+a h}\right)^{(t-s) / h}(1+c h)^{|s| / h}, \quad s \leq t, \\
& \|T(t, s) Q(s)\| \leq\left(\frac{1}{1+b h}\right)^{(s-t) / h}(1+c h)^{|s| / h}, \quad t \leq s .
\end{aligned}
$$

Example 2.4 Let $\mathbb{T}=q^{\mathbb{N} 0}, q>1$, and let the functions $a, b$ be positive constants, where $\mathbb{N}_{0}=\mathbb{N} \cup\{0\}$.

We get $\mu(t)=(q-1) t$ and $\kappa_{1}=1$. If $L_{1}\left(t_{1}, t_{2}\right)=L_{2}\left(t_{1}, t_{2}\right)=e_{c}\left(t_{1}, t_{2}\right)$, where $c$ is a positive constant, then (2.2) reduces to

$$
\begin{aligned}
& \|T(t, s) P(s)\| \leq \prod_{\tau \in[s, t)}[1 /(1+(q-1) a \tau)] \prod_{\tau \in[0, s)}[1+(q-1) c \tau], \quad s \leq t, \\
& \|T(t, s) Q(s)\| \leq \prod_{\tau \in[t, s)}[1 /(1+(q-1) b \tau)] \prod_{\tau \in[0, s)}[1+(q-1) c \tau], \quad t \leq s .
\end{aligned}
$$

\section{Parameter dependence of roughness}

The section focuses on parameter dependence of roughness for the general exponential dichotomy on time scales under the sufficiently small linear perturbation. We consider the linear perturbed system

$$
x^{\Delta}=A(t) x+B(t, \lambda) x,
$$

where $B: \mathbb{T} \times Y \rightarrow \mathscr{B}(X), Y=(Y,|\cdot|)$ is an open subset of a Banach space (the parameter space). In the rest of the section, we let $\widehat{T}_{\lambda}(t, s)$ be the evolution operator associated to system (3.1) for each $\lambda \in Y$.

To obtain our conclusion, we let

$$
\mathcal{L}:=\left\{\begin{array}{l|l}
L: \mathbb{T} \times \mathbb{T} \rightarrow \mathbb{R}^{+} & \begin{array}{l}
L(t, t)=1, L(t, s) \text { is increasing for the first } \\
\text { variable and decreasing for the second variable }
\end{array}
\end{array}\right\}
$$

and assume that the following conditions hold:

$\left(a_{1}\right)$ there exist a positive constant $c$ and a function $L^{*}: \mathbb{T} \times \mathbb{T} \rightarrow \mathbb{R}^{+}$such that

$$
\begin{aligned}
& \|B(t, \lambda)\| \leq c / L^{*}\left(\sigma(t), \kappa_{1}\right), \\
& \|B(t, \lambda)-B(t, v)\| \leq\left(c / L^{*}\left(\sigma(t), \kappa_{1}\right)\right)|\lambda-v|, \quad \kappa_{1} \leq t,
\end{aligned}
$$

and

$$
\begin{aligned}
& \|B(t, \lambda)\| \leq c / L^{*}\left(\kappa_{2}, \sigma(t)\right), \\
& \|B(t, \lambda)-B(t, v)\| \leq\left(c / L^{*}\left(\kappa_{2}, \sigma(t)\right)\right)|\lambda-v|, \quad t \leq \kappa_{2},
\end{aligned}
$$

where $\lambda, v \in \mathrm{Y}$; 
( $\left.\mathrm{a}_{2}\right)$ there exist positive constants $M_{1}, M_{2}$ such that

$$
\begin{aligned}
& \int_{\kappa_{1}}^{\infty} \frac{\max \left\{L_{1}\left(\sigma(\tau), \kappa_{1}\right), L_{2}\left(\sigma(\tau), \kappa_{1}\right)\right\} L_{2}\left(\tau, \kappa_{1}\right)}{L^{*}\left(\sigma(\tau), \kappa_{1}\right)} \Delta \tau<M_{1}, \\
& \int_{-\infty}^{\kappa_{2}} \frac{\max \left\{L_{1}\left(\kappa_{2}, \sigma(\tau)\right), L_{2}\left(\kappa_{2}, \sigma(\tau)\right)\right\} L_{2}\left(\kappa_{2}, \tau\right)}{L^{*}\left(\kappa_{2}, \sigma(\tau)\right)} \Delta \tau<M_{2} ;
\end{aligned}
$$

(a $\left.\mathrm{a}_{3}\right) \lim _{t \rightarrow-\infty} L_{1}(s, t) e_{\ominus a}(s, t)=0$ and $\lim _{t \rightarrow \infty} L_{2}(t, s) e_{\ominus b}(t, s)=0$ for each fixed $s \in \mathbb{T}$;

$\left(\mathrm{a}_{4}\right) e_{\ominus(a \oplus b)}\left(\cdot, \kappa_{1}\right) L_{2}\left(\cdot, \kappa_{1}\right)$ is a decreasing function and $e_{\ominus(a \oplus b)}\left(\kappa_{2}, \cdot\right) L_{1}\left(\kappa_{2}, \cdot\right)$ is an increasing function.

Now we state our main result in this section.

Theorem 3.1 Assume that system (2.1) admits a general exponential dichotomy on a time scale $\mathbb{T}$ with $L_{1}, L_{2} \in \mathcal{L}$ and conditions $\left(\mathrm{a}_{1}\right)-\left(\mathrm{a}_{4}\right)$ hold with sufficiently small c. Then system (3.1) also admits a general exponential dichotomy on the time scale $\mathbb{T}$, i.e., for each $\lambda \in Y$, there exist projections $\widehat{P}_{\lambda}(t)$ such that

$$
\widehat{P}_{\lambda}(t) \widehat{T}_{\lambda}(t, s)=\widehat{T}_{\lambda}(t, s) \widehat{P}_{\lambda}(s)
$$

and

$$
\begin{aligned}
& \left\|\widehat{T}_{\lambda}(t, s) \widehat{P}_{\lambda}(s)\right\| \leq\left\{\begin{array}{ll}
\frac{K \widehat{K} L_{1}\left(s, \kappa_{1}\right)\left(L_{1}\left(s, \kappa_{1}\right)+L_{2}\left(s, \kappa_{1}\right)\right)}{1-2 K \widehat{K} c\left(1+[a \mu]^{*}\right)\left(M_{1}+M_{2}\right)} e_{\ominus a}(t, s), & \kappa_{1} \leq s, \\
\frac{K \widehat{K} L_{1}\left(\kappa_{2}, s\right)\left(L_{1}\left(\kappa_{2}, s\right)+L_{2}\left(\kappa_{2}, s\right)\right)}{1-2 K \widehat{K} c\left(1+[a \mu]^{*}\right)\left(M_{1}+M_{2}\right)} e_{\ominus a}(t, s), & s \leq \kappa_{2},
\end{array} \quad s \leq t,\right. \\
& \left\|\widehat{T}_{\lambda}(t, s) \widehat{Q}_{\lambda}(s)\right\| \leq\left\{\begin{array}{lll}
\frac{K \widehat{K} L_{2}\left(s, \kappa_{1}\right)\left(L_{1}\left(s, \kappa_{1}\right)+L_{2}\left(s, \kappa_{1}\right)\right)}{1-2 K \widehat{K} c\left(1+[a \mu]^{*}\right)\left(M_{1}+M_{2}\right)} e_{\ominus b}(s, t), & \kappa_{1} \leq s, \\
\frac{K \widehat{K} L_{2}\left(\kappa_{2}, s\right)\left(L_{1}\left(\kappa_{2}, s\right)+L_{2}\left(\kappa_{2}, s\right)\right)}{1-2 K \widehat{K} c\left(1+[a \mu]^{*}\right)\left(M_{1}+M_{2}\right)} e_{\ominus b}(s, t), & s \leq \kappa_{2}, & t \leq s,
\end{array}\right.
\end{aligned}
$$

where $\widehat{Q}_{\lambda}(t)=\operatorname{Id}-\widehat{P}_{\lambda}(t)$ are the complementary projections of $\widehat{P}(t)$,

$$
\widehat{K}=K /\left(1-K c\left(\left(2+[a \mu]^{*}\right) M_{1}+\left(2+[a \mu]^{*}\right) M_{2}\right)\right) .
$$

Moreover, if $Y$ is a finite-dimensional space and $\widehat{T}_{\lambda}$ is Lipschitz continuous for the parameter $\lambda$, then the stable subspace $\widehat{P}_{\lambda}(X)$ and the unstable subspace $\widehat{Q}_{\lambda}(X)$ are Lipschitz continuous for the parameter $\lambda$.

The proof of Theorem 3.1 is nontrivial and is achieved in several steps:

(i) construct some bounded solutions of perturbed system (3.1) (Lemmas 3.1, 3.2);

(ii) semigroup properties of the bounded solutions of system (3.1) (Lemma 3.3);

(iii) construction of the projections $\widehat{P}_{\lambda}$ in (3.2) (Lemmas 3.4, 3.5 and (3.15));

(iv) norm bounds for the evolution operator $\widehat{T}_{\lambda}$ (Lemmas 3.6, 3.7, 3.8);

(v) $\widehat{P}_{\lambda}$ and $\widehat{Q}_{\lambda}$ are Lipschitz continuous for the parameter $\lambda$ (Lemma 3.9).

We set

$$
\begin{aligned}
& \Omega_{1}:=\left\{U(t, s)_{(s \leq t)} \in \mathcal{B}(X):\|U\|_{1}=\max \left\{\|U\|_{1}^{1},\|U\|_{1}^{2}\right\}<\widehat{K}\right\}, \\
& \Omega_{2}:=\left\{V(t, s)_{(t \leq s)} \in \mathcal{B}(X):\|V\|_{2}=\max \left\{\|V\|_{2}^{1},\|V\|_{2}^{2}\right\}<\widehat{K}\right\},
\end{aligned}
$$


where

$$
\begin{aligned}
& \|U\|_{1}^{1}:=\sup \left\{\|U(t, s)\| e_{a}(t, s) / L_{1}\left(s, \kappa_{1}\right): s \leq t, \kappa_{1} \leq s\right\}, \\
& \|U\|_{1}^{2}:=\sup \left\{\|U(t, s)\| e_{a}(t, s) / L_{1}\left(\kappa_{2}, s\right): s \leq t, s \leq \kappa_{2}\right\}, \\
& \|V\|_{2}^{1}:=\sup \left\{\|V(t, s)\| e_{b}(s, t) / L_{2}\left(s, \kappa_{1}\right): t \leq s, \kappa_{1} \leq s\right\}, \\
& \|V\|_{2}^{2}:=\sup \left\{\|V(t, s)\| e_{b}(s, t) / L_{2}\left(\kappa_{2}, s\right): t \leq s, s \leq \kappa_{2}\right\} .
\end{aligned}
$$

It is not difficult to show that $\left(\Omega_{1},\|\cdot\|_{1}\right)$ and $\left(\Omega_{2},\|\cdot\|_{2}\right)$ are both Banach spaces.

Lemma 3.1 For each $\lambda \in Y$, there exists a unique bounded solution $U_{\lambda} \in \Omega_{1}$ satisfying

$$
\begin{aligned}
U_{\lambda}(t, s)= & T(t, s) P(s)+\int_{s}^{t} T(t, \sigma(\tau)) P(\sigma(\tau)) B(\tau, \lambda) U_{\lambda}(\tau, s) \Delta \tau \\
& -\int_{t}^{\infty} T(t, \sigma(\tau)) Q(\sigma(\tau)) B(\tau, \lambda) U_{\lambda}(\tau, s) \Delta \tau, \quad s \leq t
\end{aligned}
$$

and $U_{\lambda}$ is Lipschitz continuous for the parameter $\lambda$.

Proof Direct calculation shows that $U_{\lambda}$ satisfying (3.6) is a solution of (3.1). For each $\lambda \in Y$, we define an operator $J^{\lambda}$ on $\Omega_{1}$ by

$$
\begin{aligned}
\left(J^{\lambda} U\right)(t, s)= & T(t, s) P(s)+\int_{s}^{t} T(t, \sigma(\tau)) P(\sigma(\tau)) B(\tau, \lambda) U(\tau, s) \Delta \tau \\
& -\int_{t}^{\infty} T(t, \sigma(\tau)) Q(\sigma(\tau)) B(\tau, \lambda) U(\tau, s) \Delta \tau .
\end{aligned}
$$

By (2.2), (2.3), $\left(\mathrm{a}_{1}\right)$ and $\left(\mathrm{a}_{2}\right)$, we have

$$
\begin{aligned}
\left\|\left(J^{\lambda} U\right)(t, s)\right\| \leq & K L_{1}\left(s, \kappa_{1}\right) e_{\ominus a}(t, s)+K c \int_{s}^{t} \frac{L_{1}\left(\sigma(\tau), \kappa_{1}\right) e_{\ominus a}(t, \sigma(\tau))}{L^{*}\left(\sigma(\tau), \kappa_{1}\right)}\|U(\tau, s)\| \Delta \tau \\
& +K c \int_{t}^{\infty} \frac{L_{2}\left(\sigma(\tau), \kappa_{1}\right) e_{\ominus b}(\sigma(\tau), t)}{L^{*}\left(\sigma(\tau), \kappa_{1}\right)}\|U(\tau, s)\| \Delta \tau \\
\leq & K L_{1}\left(s, \kappa_{1}\right) e_{\ominus a}(t, s) \\
& +K c \int_{s}^{t} \frac{L_{1}\left(\sigma(\tau), \kappa_{1}\right) e_{\ominus a}(t, \sigma(\tau))}{L^{*}\left(\sigma(\tau), \kappa_{1}\right)} e_{\ominus a}(\tau, s) L_{1}\left(s, \kappa_{1}\right) \Delta \tau\|U\|_{1}^{1} \\
& +K c \int_{t}^{\infty} \frac{L_{2}\left(\sigma(\tau), \kappa_{1}\right) e_{\ominus b}(\sigma(\tau), t)}{L^{*}\left(\sigma(\tau), \kappa_{1}\right)} e_{\ominus a}(\tau, s) L_{1}\left(s, \kappa_{1}\right) \Delta \tau\|U\|_{1}^{1} \\
\leq & K L_{1}\left(s, \kappa_{1}\right) e_{\ominus a}(t, s)\left(1+c\|U\|_{1}^{1} \int_{s}^{t}(1+a \mu(\tau)) \frac{L_{1}\left(\sigma(\tau), \kappa_{1}\right)}{L^{*}\left(\sigma(\tau), \kappa_{1}\right)} \Delta \tau\right. \\
& \left.+c\|U\|_{1}^{1} \int_{t}^{\infty} \frac{1}{1+b \mu(\tau)} \frac{L_{2}\left(\sigma(\tau), \kappa_{1}\right)}{L^{*}\left(\sigma(\tau), \kappa_{1}\right)} \Delta \tau\right) \\
\leq & K L_{1}\left(s, \kappa_{1}\right) e_{\ominus a}(t, s)\left(1+c\left(2+[a \mu]^{*}\right) M_{1}\|U\|_{1}^{1}\right)
\end{aligned}
$$


for $\kappa_{1} \leq s$ and

$$
\begin{aligned}
\left\|\left(J^{\lambda} U\right)(t, s)\right\| \leq & K L_{1}\left(\kappa_{2}, s\right) e_{\ominus a}(t, s)+K c \int_{s}^{t} \frac{L_{1}\left(\kappa_{2}, \sigma(\tau)\right) e_{\ominus a}(t, \sigma(\tau))}{L^{*}\left(\kappa_{2}, \sigma(\tau)\right)}\|U(\tau, s)\| \Delta \tau \\
& +K c \int_{t}^{\kappa_{2}} \frac{L_{2}\left(\kappa_{2}, \sigma(\tau)\right) e_{\ominus b}(\sigma(\tau), t)}{L^{*}\left(\kappa_{2}, \sigma(\tau)\right)}\|U(\tau, s)\| \Delta \tau \\
& +K c \int_{\kappa_{1}}^{\infty} \frac{L_{2}\left(\sigma(\tau), \kappa_{1}\right) e_{\ominus b}(\sigma(\tau), t)}{L^{*}\left(\sigma(\tau), \kappa_{1}\right)}\|U(\tau, s)\| \Delta \tau \\
\leq & K L_{1}\left(\kappa_{2}, s\right) e_{\ominus a}(t, s) \\
& +K c \int_{s}^{t} \frac{L_{1}\left(\kappa_{2}, \sigma(\tau)\right) e_{\ominus a}(t, \sigma(\tau))}{L^{*}\left(\kappa_{2}, \sigma(\tau)\right)} e_{\ominus a}(\tau, s) L_{1}\left(\kappa_{2}, s\right) \Delta \tau\|U\|_{1}^{2} \\
& +K c \int_{t}^{\kappa_{2}} \frac{L_{2}\left(\kappa_{2}, \sigma(\tau)\right) e_{\ominus b}(\sigma(\tau), t)}{L^{*}\left(\kappa_{2}, \sigma(\tau)\right)} e_{\ominus a}(\tau, s) L_{1}\left(\kappa_{2}, s\right) \Delta \tau\|U\|_{1}^{2} \\
& +K c \int_{\kappa_{1}}^{\infty} \frac{L_{2}\left(\sigma(\tau), \kappa_{1}\right) e_{\ominus b}(\sigma(\tau), t)}{L^{*}\left(\sigma(\tau), \kappa_{1}\right)} e_{\ominus a}(\tau, s) L_{1}\left(\kappa_{2}, s\right) \Delta \tau\|U\|_{1}^{2} \\
\leq & K L_{1}\left(\kappa_{2}, s\right) e_{\ominus a}(t, s)\left(1+c\|U\|_{1}^{2} \int_{s}^{t}(1+a \mu(\tau)) \frac{L_{1}\left(\kappa_{2}, \sigma(\tau)\right)}{L^{*}\left(\kappa_{2}, \sigma(\tau)\right)} \Delta \tau\right. \\
& +c\|U\|_{1}^{2} \int_{t}^{\kappa_{2}} \frac{1}{1+b \mu(\tau)} \frac{L_{2}\left(\kappa_{2}, \sigma(\tau)\right)}{L^{*}\left(\kappa_{2}, \sigma(\tau)\right)} \Delta \tau \\
& \left.+c\|U\|_{1}^{2} \int_{\kappa_{1}}^{\infty} \frac{1}{1+b \mu(\tau)} \frac{L_{2}\left(\sigma(\tau), \kappa_{1}\right)}{L^{*}\left(\sigma(\tau), \kappa_{1}\right)} \Delta \tau\right) \\
\leq & K L_{1}\left(\kappa_{2}, s\right) e_{\ominus a}(t, s)\left(1+c\left(M_{1}+\left(2+[a \mu]^{*}\right) M_{2}\right)\|U\|_{1}^{2}\right)
\end{aligned}
$$

for $t \leq \kappa_{2}$. For $s \leq \kappa_{2} \leq \kappa_{1} \leq t$, we get

$$
\begin{aligned}
\left\|\left(J^{\lambda} U\right)(t, s)\right\| \leq & K L_{1}\left(\kappa_{2}, s\right) e_{\ominus a}(t, s)+K c \int_{s}^{\kappa_{2}} \frac{L_{1}\left(\kappa_{2}, \sigma(\tau)\right) e_{\ominus a}(t, \sigma(\tau))}{L^{*}\left(\kappa_{2}, \sigma(\tau)\right)}\|U(\tau, s)\| \Delta \tau \\
& +K c \int_{\kappa_{1}}^{t} \frac{L_{2}\left(\sigma(\tau), \kappa_{1}\right) e_{\ominus a}(t, \sigma(\tau))}{L^{*}\left(\sigma(\tau), \kappa_{1}\right)}\|U(\tau, s)\| \Delta \tau \\
& +K c \int_{t}^{\infty} \frac{L_{2}\left(\sigma(\tau), \kappa_{1}\right) e_{\ominus b}(\sigma(\tau), t)}{L^{*}\left(\sigma(\tau), \kappa_{1}\right)}\|U(\tau, s)\| \Delta \tau \\
\leq & K L_{1}\left(\kappa_{2}, s\right) e_{\ominus a}(t, s) \\
& +K c \int_{s}^{\kappa_{2}} \frac{L_{1}\left(\kappa_{2}, \sigma(\tau)\right) e_{\ominus a}(t, \sigma(\tau))}{L^{*}\left(\kappa_{2}, \sigma(\tau)\right)} e_{\ominus a}(\tau, s) L_{1}\left(\kappa_{2}, s\right) \Delta \tau\|U\|_{1}^{2} \\
& +K c \int_{\kappa_{1}}^{t} \frac{L_{1}\left(\sigma(\tau), \kappa_{1}\right) e_{\ominus a}(t, \sigma(\tau))}{L^{*}\left(\sigma(\tau), \kappa_{1}\right)} e_{\ominus a}(\tau, s) L_{1}\left(\kappa_{2}, s\right) \Delta \tau\|U\|_{1}^{2} \\
& +K c \int_{t}^{\infty} \frac{L_{2}\left(\sigma(\tau), \kappa_{1}\right) e_{\ominus b}(\sigma(\tau), t)}{L^{*}\left(\sigma(\tau), \kappa_{1}\right)} e_{\ominus a}(\tau, s) L_{1}\left(\kappa_{2}, s\right) \Delta \tau\|U\|_{1}^{2} \\
\leq & K L_{1}\left(\kappa_{2}, s\right) e_{\ominus a}(t, s)\left(1+c\|U\|_{1}^{2} \int_{s}^{\kappa_{2}}(1+a \mu(\tau)) \frac{L_{1}\left(\kappa_{2}, \sigma(\tau)\right)}{L^{*}\left(\kappa_{2}, \sigma(\tau)\right)} \Delta \tau\right. \\
& +c\|U\|_{1}^{2} \int_{\kappa_{1}}^{t}(1+a \mu(\tau)) \frac{L_{1}\left(\sigma(\tau), \kappa_{1}\right)}{L^{*}\left(\sigma(\tau), \kappa_{1}\right)} \Delta \tau
\end{aligned}
$$




$$
\begin{aligned}
& \left.+c\|U\|_{1}^{2} \int_{t}^{\infty} \frac{1}{1+b \mu(\tau)} \frac{L_{2}\left(\sigma(\tau), \kappa_{1}\right)}{L^{*}\left(\sigma(\tau), \kappa_{1}\right)} \Delta \tau\right) \\
\leq & K L_{1}\left(\kappa_{2}, s\right) e_{\ominus a}(t, s)\left(1+c\left(\left(2+[a \mu]^{*}\right) M_{1}+\left(1+[a \mu]^{*}\right) M_{2}\right)\|U\|_{1}^{2}\right) .
\end{aligned}
$$

Then

$$
\left\|J^{\lambda} U\right\|_{1}^{1} \leq K\left(1+c\left(2+[a \mu]^{*}\right) M_{1}\|U\|_{1}^{1}\right) \leq \widehat{K}
$$

and

$$
\left\|J^{\lambda} U\right\|_{1}^{2} \leq K\left(1+c\left(\left(2+[a \mu]^{*}\right) M_{1}+\left(2+[a \mu]^{*}\right) M_{2}\right)\|U\|_{1}^{2}\right) \leq \widehat{K},
$$

that is,

$$
\left\|J^{\lambda} U\right\|_{1} \leq \max \left\{\left\|\left(J^{\lambda} U\right)\right\|_{1}^{1},\left\|\left(J^{\lambda} U\right)\right\|_{1}^{2}\right\} \leq \widehat{K} .
$$

This implies that $J^{\lambda}\left(\Omega_{1}\right) \subset \Omega_{1}$. Similarly, for each $U_{1}, U_{2} \in \Omega_{1}$, we get

$$
\left\|J^{\lambda} U\right\|_{1} \leq K c\left(\left(2+[a \mu]^{*}\right) M_{1}+\left(2+[a \mu]^{*}\right) M_{2}\right)\left\|U_{1}-U_{2}\right\|_{1} .
$$

If $c$ is sufficiently small, then $J^{\lambda}$ is a contraction and for each $\lambda \in Y$, there exists a unique $U_{\lambda} \in \Omega_{1}$ such that $J^{\lambda} U_{\lambda}=U_{\lambda}$ and (3.6) holds.

Next we show that $U_{\lambda}$ is Lipschitz continuous for the parameter $\lambda$. For any $\lambda_{1}, \lambda_{2} \in Y$, there exist bounded solutions $U_{\lambda_{1}}, U_{\lambda_{2}} \in \Omega_{1}$ satisfying (3.6). It follows from $\left(\mathrm{a}_{2}\right)$ that

$$
\begin{aligned}
A_{1}(\tau) & :=\left\|B\left(\tau, \lambda_{1}\right) U_{\lambda_{1}}(\tau, s)-B\left(\tau, \lambda_{2}\right) U_{\lambda_{2}}(\tau, s)\right\| \\
& \leq\left\|B\left(\tau, \lambda_{1}\right) U_{\lambda_{1}}(\tau, s)-B\left(\tau, \lambda_{1}\right) U_{\lambda_{2}}(\tau, s)\right\|+\left\|B\left(\tau, \lambda_{1}\right) U_{\lambda_{2}}(\tau, s)-B\left(\tau, \lambda_{2}\right) U_{\lambda_{2}}(\tau, s)\right\| \\
& \leq \frac{c e_{\ominus a}(\tau, s) L_{1}\left(s, \kappa_{1}\right)}{L^{*}\left(\sigma(\tau), \kappa_{1}\right)}\left\|U_{\lambda_{1}}-U_{\lambda_{2}}\right\|_{1}^{1}+\frac{\widehat{K} c e_{\ominus a}(\tau, s) L_{1}\left(s, \kappa_{1}\right)\left|\lambda_{1}-\lambda_{2}\right|}{L^{*}\left(\sigma(\tau), \kappa_{1}\right)}
\end{aligned}
$$

for $\tau \geq s \geq \kappa_{1}$ and

$$
\begin{aligned}
A_{2}(\tau) & :=\left\|B\left(\tau, \lambda_{1}\right) U_{\lambda_{1}}(\tau, s)-B\left(\tau, \lambda_{2}\right) U_{\lambda_{2}}(\tau, s)\right\| \\
& \leq\left\|B\left(\tau, \lambda_{1}\right) U_{\lambda_{1}}(\tau, s)-B\left(\tau, \lambda_{1}\right) U_{\lambda_{2}}(\tau, s)\right\|+\left\|B\left(\tau, \lambda_{1}\right) U_{\lambda_{2}}(\tau, s)-B\left(\tau, \lambda_{2}\right) U_{\lambda_{2}}(\tau, s)\right\| \\
& \leq \frac{c e_{\ominus a}(\tau, s) L_{1}\left(\kappa_{2}, s\right)}{L^{*}\left(\kappa_{2}, \sigma(\tau)\right)}\left\|U_{\lambda_{1}}-U_{\lambda_{2}}\right\|_{1}^{2}+\frac{\widehat{K} c e_{\ominus a}(\tau, s) L_{1}\left(\kappa_{2}, s\right)\left|\lambda_{1}-\lambda_{2}\right|}{L^{*}\left(\kappa_{2}, \sigma(\tau)\right)}
\end{aligned}
$$

for $s \leq \tau \leq \kappa_{2}$. Moreover, we also have

$$
\begin{aligned}
A_{3}(\tau) & :=\left\|B\left(\tau, \lambda_{1}\right) U_{\lambda_{1}}(\tau, s)-B\left(\tau, \lambda_{2}\right) U_{\lambda_{2}}(\tau, s)\right\| \\
& \leq\left\|B\left(\tau, \lambda_{1}\right) U_{\lambda_{1}}(\tau, s)-B\left(\tau, \lambda_{1}\right) U_{\lambda_{2}}(\tau, s)\right\|+\left\|B\left(\tau, \lambda_{1}\right) U_{\lambda_{2}}(\tau, s)-B\left(\tau, \lambda_{2}\right) U_{\lambda_{2}}(\tau, s)\right\| \\
& \leq \frac{c e_{\ominus a}(\tau, s) L_{1}\left(\kappa_{2}, s\right)}{L^{*}\left(\sigma(\tau), \kappa_{1}\right)}\left\|U_{\lambda_{1}}-U_{\lambda_{2}}\right\|_{1}^{2}+\frac{\widehat{K} c e_{\ominus a}(\tau, s) L_{1}\left(\kappa_{2}, s\right)\left|\lambda_{1}-\lambda_{2}\right|}{L^{*}\left(\sigma(\tau), \kappa_{1}\right)}
\end{aligned}
$$


for $s \leq \kappa_{2} \leq \kappa_{1} \leq \tau$. It follows from (2.2), (2.3), $\left(\mathrm{a}_{1}\right)$ and $\left(\mathrm{a}_{2}\right)$ that

$$
\begin{aligned}
\left\|U_{\lambda_{1}}(t, s)-U_{\lambda_{2}}(t, s)\right\| \leq & \int_{s}^{t}\|T(t, \sigma(\tau)) P(\sigma(\tau))\| A_{1}(\tau) \Delta \tau \\
& +\int_{t}^{\infty}\|T(t, \sigma(\tau)) Q(\sigma(\tau))\| A_{1}(\tau) \Delta \tau \\
\leq & K c L_{1}\left(s, \kappa_{1}\right) e_{\ominus a}(t, s)\left(2+[a \mu]^{*}\right) M_{1}\left(\left\|U_{\lambda_{1}}-U_{\lambda_{2}}\right\|_{1}^{1}+\widehat{K}\left|\lambda_{1}-\lambda_{2}\right|\right)
\end{aligned}
$$

for $s \geq \kappa_{1}$ and

$$
\begin{aligned}
\left\|U_{\lambda_{1}}(t, s)-U_{\lambda_{2}}(t, s)\right\| \leq & \int_{s}^{t}\|T(t, \sigma(\tau)) P(\sigma(\tau))\| A_{2}(\tau) \Delta \tau \\
& +\int_{t}^{\kappa 2}\|T(t, \sigma(\tau)) Q(\sigma(\tau))\| A_{2}(\tau) \Delta \tau \\
& +\int_{\kappa_{1}}^{\infty}\|T(t, \sigma(\tau)) Q(\sigma(\tau))\| A_{3}(\tau) \Delta \tau \\
\leq & K c L_{1}\left(\kappa_{2}, s\right) e_{\ominus a}(t, s)\left(M_{1}+\left(2+[a \mu]^{*}\right) M_{2}\right) \\
& \times\left(\left\|U_{\lambda_{1}}-U_{\lambda_{2}}\right\|_{1}^{2}+\widehat{K}\left|\lambda_{1}-\lambda_{2}\right|\right)
\end{aligned}
$$

for $t \leq \kappa_{2}$. For $s \leq \kappa_{2} \leq \kappa_{1} \leq t$, we get

$$
\begin{aligned}
\left\|U_{\lambda_{1}}(t, s)-U_{\lambda_{2}}(t, s)\right\| \leq & \int_{s}^{\kappa_{2}}\|T(t, \sigma(\tau)) P(\sigma(\tau))\| A_{2}(\tau) \Delta \tau \\
& +\int_{\kappa_{1}}^{t}\|T(t, \sigma(\tau)) P(\sigma(\tau))\| A_{3}(\tau) \Delta \tau \\
& +\int_{t}^{\infty}\|T(t, \sigma(\tau)) Q(\sigma(\tau))\| A_{3}(\tau) \Delta \tau \\
\leq & K c L_{1}\left(\kappa_{2}, s\right) e_{\ominus a}(t, s)\left(\left(2+[a \mu]^{*}\right) M_{1}+\left(1+[a \mu]^{*}\right) M_{2}\right) \\
& \times\left(\left\|U_{\lambda_{1}}-U_{\lambda_{2}}\right\|_{1}^{2}+\widehat{K}\left|\lambda_{1}-\lambda_{2}\right|\right) .
\end{aligned}
$$

Then

$$
\left\|U_{\lambda_{1}}-U_{\lambda_{2}}\right\|_{1} \leq \widehat{K}^{2} c\left(\left(2+[a \mu]^{*}\right) M_{1}+\left(2+[a \mu]^{*}\right) M_{2}\right)\left|\lambda_{1}-\lambda_{2}\right| .
$$

The proof of the lemma is complete.

Similarly, we have the following lemma.

Lemma 3.2 For each $\lambda \in Y$, there exists a unique bounded solution $V_{\lambda} \in \Omega_{2}$ satisfying

$$
\begin{aligned}
V_{\lambda}(t, s)= & T(t, s) Q(s)+\int_{-\infty}^{t} T(t, \sigma(\tau)) P(\sigma(\tau)) B(\tau, \lambda) V_{\lambda}(\tau, s) \Delta \tau \\
& -\int_{t}^{s} T(t, \sigma(\tau)) Q(\sigma(\tau)) B(\tau, \lambda) V_{\lambda}(\tau, s) \Delta \tau, \quad t \leq s
\end{aligned}
$$

and $V_{\lambda}$ is Lipschitz continuous for the parameter $\lambda$. 
Lemma 3.3 For each $\lambda \in Y$, the bounded solutions $U_{\lambda}$ and $V_{\lambda}$ of system (3.1) satisfy

$$
\begin{array}{ll}
U_{\lambda}(t, l) U_{\lambda}(l, s)=U_{\lambda}(t, s), & s \leq l \leq t \\
V_{\lambda}(t, l) V_{\lambda}(l, s)=V_{\lambda}(t, s), & t \leq l \leq s .
\end{array}
$$

Proof From (3.6), we get

$$
\begin{aligned}
U_{\lambda}(t, l) U_{\lambda}(l, s)= & T(t, s) P(s)+\int_{s}^{l} T(t, \sigma(\tau)) P(\sigma(\tau)) B(\tau, \lambda) U_{\lambda}(\tau, s) \Delta \tau \\
& +\int_{l}^{t} T(t, \sigma(\tau)) P(\sigma(\tau)) B(\tau, \lambda) U_{\lambda}(\tau, l) \Delta \tau U_{\lambda}(l, s) \\
& -\int_{t}^{\infty} T(t, \sigma(\tau)) Q(\sigma(\tau)) B(\tau, \lambda) U_{\lambda}(\tau, l) \Delta \tau U_{\lambda}(l, s)
\end{aligned}
$$

for $s \leq l \leq t$, and let $H_{\lambda}(t, l)=U_{\lambda}(t, l) U_{\lambda}(l, s)-U_{\lambda}(t, s)$. We define the operator $L^{\lambda}$ by

$$
\begin{aligned}
\left(L^{\lambda} h\right)(t, l)= & \int_{l}^{t} T(t, \sigma(\tau)) P(\sigma(\tau)) B(\tau, \lambda) h(\tau, l) \Delta \tau \\
& -\int_{t}^{\infty} T(t, \sigma(\tau)) Q(\sigma(\tau)) B(\tau, \lambda) h(\tau, l) \Delta \tau
\end{aligned}
$$

for any $h \in \Omega_{1}^{l}$ and each $\lambda \in Y$, where $\Omega_{1}^{l}$ is obtained from $\Omega_{1}$ replacing $s$ by $l$. Obviously, $L^{\lambda} H_{\lambda}=H_{\lambda}$. Carrying out similar arguments to the proof of Lemma 3.1, we have

$$
\left\|L^{\lambda} h\right\|_{1} \leq K c\left(\left(2+[a \mu]^{*}\right) M_{1}+\left(2+[a \mu]^{*}\right) M_{2}\right)\|h\|_{1} \leq \widehat{K}
$$

and

$$
\left\|L^{\lambda} h_{1}-L^{\lambda} h_{2}\right\|_{1} \leq K c\left(\left(2+[a \mu]^{*}\right) M_{1}+\left(2+[a \mu]^{*}\right) M_{2}\right)\left\|h_{1}-h_{2}\right\|_{1}
$$

for any $h, h_{1}, h_{2} \in \Omega_{1}^{l}$. This means that there exists a unique $h_{\lambda} \in \Omega_{1}^{l}$ such that $L h_{\lambda}=h_{\lambda}$. On the other hand, we also note that $0 \in \Omega_{1}^{l}$ and $L^{\lambda} 0=0$. Therefore, $H_{\lambda}=h_{\lambda}=0$. Similarly, the second identity of (3.9) holds.

Now we construct the projections $\widehat{P}_{\lambda}(t)$ for each $\lambda \in Y$. We first set

$$
\begin{aligned}
& \widetilde{P}_{\lambda}(t)=\widehat{T}_{\lambda}\left(t, \kappa_{1}\right) U_{\lambda}\left(\kappa_{1}, \kappa_{1}\right) \widehat{T}_{\lambda}\left(\kappa_{1}, t\right), \\
& \widetilde{Q}_{\lambda}(t)=\widehat{T}_{\lambda}\left(t, \kappa_{1}\right) V_{\lambda}\left(\kappa_{1}, \kappa_{1}\right) \widehat{T}_{\lambda}\left(\kappa_{1}, t\right) .
\end{aligned}
$$

Lemma 3.4 For each $\lambda \in Y$, we have

(b $\left.\mathrm{b}_{1}\right) \widetilde{P}_{\lambda}(t), \widetilde{Q}_{\lambda}(t)$ are projections for each $t \in \mathbb{T}$ and $\lambda \in Y$;

$\left(\mathrm{b}_{2}\right) \widetilde{P}_{\lambda}(t) \widehat{T}_{\lambda}(t, s)=\widehat{T}_{\lambda}(t, s) \widetilde{P}_{\lambda}(s), \widetilde{Q}_{\lambda}(t) \widehat{T}_{\lambda}(t, s)=\widehat{T}_{\lambda}(t, s) \widetilde{Q}_{\lambda}(s), t, s \in \mathbb{T} ;$

$\left(\mathrm{b}_{3}\right) P\left(\kappa_{1}\right) \widetilde{P}_{\lambda}\left(\kappa_{1}\right)=P\left(\kappa_{1}\right), Q\left(\kappa_{1}\right) \widetilde{Q}_{\lambda}\left(\kappa_{1}\right)=Q\left(\kappa_{1}\right), Q\left(\kappa_{1}\right)\left(\operatorname{Id}-\widetilde{P}_{\lambda}\left(\kappa_{1}\right)\right)=\operatorname{Id}-\widetilde{P}_{\lambda}\left(\kappa_{1}\right), P\left(\kappa_{1}\right)(\operatorname{Id}-$ $\left.\widetilde{Q}_{\lambda}\left(\kappa_{1}\right)\right)=\mathrm{Id}-\widetilde{Q}_{\lambda}\left(\kappa_{1}\right)$

(b $\left.\mathrm{b}_{4}\right) \widetilde{P}_{\lambda}\left(\kappa_{1}\right) P\left(\kappa_{1}\right)=\widetilde{P}_{\lambda}\left(\kappa_{1}\right), \widetilde{Q}_{\lambda}\left(\kappa_{1}\right) Q\left(\kappa_{1}\right)=\widetilde{Q}_{\lambda}\left(\kappa_{1}\right)$. 
Proof It follows from Lemma 3.3 that $\left(b_{1}\right)$ and $\left(b_{2}\right)$ hold. By (3.6) and (3.8), we get

$$
\begin{aligned}
& \widetilde{P}_{\lambda}\left(\kappa_{1}\right)=U_{\lambda}\left(\kappa_{1}, \kappa_{1}\right)=P\left(\kappa_{1}\right)-\int_{\kappa_{1}}^{\infty} T\left(\kappa_{1}, \sigma(\tau)\right) Q(\sigma(\tau)) B(\tau, \lambda) U_{\lambda}\left(\tau, \kappa_{1}\right) \Delta \tau, \\
& \widetilde{Q}_{\lambda}\left(\kappa_{1}\right)=V_{\lambda}\left(\kappa_{1}, \kappa_{1}\right)=Q\left(\kappa_{1}\right)+\int_{-\infty}^{\kappa_{1}} T\left(\kappa_{1}, \sigma(\tau)\right) P(\sigma(\tau)) B(\tau, \lambda) V_{\lambda}\left(\tau, \kappa_{1}\right) \Delta \tau,
\end{aligned}
$$

which imply that $\left(\mathrm{b}_{3}\right)$ holds. By Lemma 3.1 and Lemma 3.2, we have $U_{\lambda}\left(t, \kappa_{1}\right) P\left(\kappa_{1}\right)=$ $U_{\lambda}\left(t, \kappa_{1}\right)$ and $V_{\lambda}\left(t, \kappa_{1}\right) Q\left(\kappa_{1}\right)=V_{\lambda}\left(t, \kappa_{1}\right)$ since $U_{\lambda}\left(t, \kappa_{1}\right) P\left(\kappa_{1}\right) \in \Omega_{1}$ satisfies identity (3.6) with $s=\kappa_{1}$ and $V_{\lambda}\left(t, \kappa_{1}\right) Q\left(\kappa_{1}\right) \in \Omega_{2}$ satisfies identity (3.8) with $s=\kappa_{1}$. Therefore, $\left(\mathrm{b}_{4}\right)$ holds.

Lemma 3.5 For each $\lambda \in Y, S_{\lambda}\left(\kappa_{1}\right)=\widetilde{P}_{\lambda}\left(\kappa_{1}\right)+\widetilde{Q}_{\lambda}\left(\kappa_{1}\right)$ is invertible.

Proof For each $\lambda \in Y$, combining $\left(\mathrm{b}_{3}\right)$ and $\left(\mathrm{b}_{4}\right)$ together gives

$$
\widetilde{P}_{\lambda}\left(\kappa_{1}\right)+\widetilde{Q}_{\lambda}\left(\kappa_{1}\right)-\mathrm{Id}=Q\left(\kappa_{1}\right) \widetilde{P}_{\lambda}\left(\kappa_{1}\right)+P\left(\kappa_{1}\right) \widetilde{Q}_{\lambda}\left(\kappa_{1}\right)
$$

It follows from (3.11) and (3.12) that

$$
\begin{aligned}
& P\left(\kappa_{1}\right) \widetilde{Q}_{\lambda}\left(\kappa_{1}\right)=P\left(\kappa_{1}\right) V_{\lambda}\left(\kappa_{1}, \kappa_{1}\right)=\int_{-\infty}^{\kappa_{1}} T\left(\kappa_{1}, \sigma(\tau)\right) P(\sigma(\tau)) B(\tau, \lambda) V_{\lambda}\left(\tau, \kappa_{1}\right) \Delta \tau, \\
& Q\left(\kappa_{1}\right) \widetilde{P}_{\lambda}\left(\kappa_{1}\right)=Q\left(\kappa_{1}\right) U_{\lambda}\left(\kappa_{1}, \kappa_{1}\right)=-\int_{\kappa_{1}}^{\infty} T\left(\kappa_{1}, \sigma(\tau)\right) Q(\sigma(\tau)) B(\tau, \lambda) U_{\lambda}\left(\tau, \kappa_{1}\right) \Delta \tau .
\end{aligned}
$$

Note that

$$
\begin{gathered}
\left\|U_{\lambda}(t, s)\right\| \leq \widehat{K} L_{1}\left(s, \kappa_{1}\right) e_{\ominus a}(t, s), \quad s \leq t, \kappa_{1} \leq s, \\
\left\|U_{\lambda}(t, s)\right\| \leq \widehat{K} L_{1}\left(\kappa_{2}, s\right) e_{\ominus a}(t, s), \quad s \leq t, s \leq \kappa_{2}
\end{gathered}
$$

and

$$
\begin{gathered}
\left\|V_{\lambda}(t, s)\right\| \leq \widehat{K} L_{2}\left(s, \kappa_{1}\right) e_{\ominus b}(s, t), \quad t \leq s, \kappa_{1} \leq s \\
\left\|V_{\lambda}(t, s)\right\| \leq \widehat{K} L_{2}\left(\kappa_{2}, s\right) e_{\ominus b}(s, t), \quad t \leq s, s \leq \kappa_{2} .
\end{gathered}
$$

Then

$$
\begin{aligned}
& \int_{-\infty}^{\kappa_{1}}\left\|T\left(\kappa_{1}, \sigma(\tau)\right) P(\sigma(\tau))\right\|\|B(\tau, \lambda)\|\left\|V_{\lambda}\left(\tau, \kappa_{1}\right)\right\| \Delta \tau \\
& \quad=\int_{-\infty}^{\kappa_{2}}\left\|T\left(\kappa_{1}, \sigma(\tau)\right) P(\sigma(\tau))\right\|\|B(\tau, \lambda)\|\left\|V_{\lambda}\left(\tau, \kappa_{1}\right)\right\| \Delta \tau \\
& \quad \leq K \widehat{K} \int_{-\infty}^{\kappa_{2}} L_{1}\left(\kappa_{2}, \sigma(\tau)\right) e_{\ominus a}\left(\kappa_{1}, \sigma(\tau)\right)\left(c / L^{*}\left(\kappa_{2}, \sigma(\tau)\right)\right) L_{2}\left(\kappa_{1}, \kappa_{1}\right) e_{\ominus b}\left(\kappa_{1}, \tau\right) \Delta \tau \\
& \leq K \widehat{K} c \int_{-\infty}^{\kappa_{2}} \frac{L_{1}\left(\kappa_{2}, \sigma(\tau)\right)}{L^{*}\left(\kappa_{2}, \sigma(\tau)\right)} \Delta \tau \leq K \widehat{K} c M_{2}
\end{aligned}
$$


and

$$
\begin{aligned}
& \int_{\kappa_{1}}^{\infty}\left\|T\left(\kappa_{1}, \sigma(\tau)\right) Q(\sigma(\tau))\right\|\|B(\tau, \lambda)\|\left\|U_{\lambda}\left(\tau, \kappa_{1}\right)\right\| \Delta \tau \\
& \quad \leq K \widehat{K} \int_{\kappa_{1}}^{\infty} L_{2}\left(\sigma(\tau), \kappa_{1}\right) e_{\ominus b}\left(\sigma(\tau), \kappa_{1}\right)\left(c / L^{*}\left(\sigma(\tau), \kappa_{1}\right)\right) L_{1}\left(\kappa_{1}, \kappa_{1}\right) e_{\ominus a}\left(\tau, \kappa_{1}\right) \Delta \tau \\
& \quad \leq K \widehat{K} c \int_{\kappa_{1}}^{\infty} \frac{L_{2}\left(\sigma(\tau), \kappa_{1}\right)}{L^{*}\left(\sigma(\tau), \kappa_{1}\right)} \Delta \tau \leq K \widehat{K} c M_{1} .
\end{aligned}
$$

Then

$$
\left\|\widetilde{P}_{\lambda}\left(\kappa_{1}\right)+\widetilde{Q}_{\lambda}\left(\kappa_{1}\right)-\operatorname{Id}\right\| \leq K \widehat{K} c\left(M_{1}+M_{2}\right)
$$

which means that $S_{\lambda}\left(\kappa_{1}\right)$ is invertible if $c$ is sufficiently small.

We set

$$
\begin{aligned}
& \widehat{P}_{\lambda}(t)=\widehat{T}_{\lambda}\left(t, \kappa_{1}\right) S_{\lambda}\left(\kappa_{1}\right) P\left(\kappa_{1}\right) S_{\lambda}^{-1}\left(\kappa_{1}\right) \widehat{T}_{\lambda}\left(\kappa_{1}, t\right), \\
& \widehat{Q}_{\lambda}(t)=\widehat{T}_{\lambda}\left(t, \kappa_{1}\right) S_{\lambda}\left(\kappa_{1}\right) Q\left(\kappa_{1}\right) S_{\lambda}^{-1}\left(\kappa_{1}\right) \widehat{T}_{\lambda}\left(\kappa_{1}, t\right)
\end{aligned}
$$

for each $\lambda \in Y$ and $t \in \mathbb{T}$. It is not difficult to show that $\widehat{P}_{\lambda}(t)+\widehat{Q}_{\lambda}(t)=\mathrm{Id}, \lambda \in Y, t \in \mathbb{T}$. Then $\widehat{P}_{\lambda}(t), \widehat{Q}_{\lambda}(t)$ are projections and (3.2) is valid.

Lemma 3.6 We have

$$
\begin{aligned}
& \left\|\widehat{T}_{\lambda}(t, s) \mid \operatorname{Im} \widetilde{P}_{\lambda}(s)\right\| \leq\left\{\begin{array}{ll}
\widehat{K} L_{1}\left(s, \kappa_{1}\right) e_{\ominus a}(t, s), \quad \kappa_{1} \leq s, \\
\widehat{K} L_{1}\left(\kappa_{2}, s\right) e_{\ominus a}(t, s), \quad s \leq \kappa_{2},
\end{array} \quad s \leq t,\right.
\end{aligned}
$$

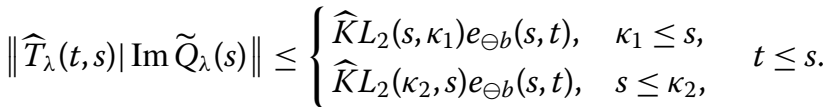

Proof We first prove that for each $\lambda \in Y$, if $z_{\lambda}(t)_{(t \geq s)}$ is a bounded solution of (3.1), then

$$
\begin{aligned}
z_{\lambda}(t)= & T(t, s) P(s) z_{\lambda}(s)+\int_{s}^{t} T(t, \sigma(\tau)) P(\sigma(\tau)) B(\tau, \lambda) z_{\lambda}(\tau) \Delta \tau \\
& -\int_{t}^{\infty} T(t, \sigma(\tau)) Q(\sigma(\tau)) B(\tau, \lambda) z_{\lambda}(\tau) \Delta \tau, \quad s \leq t .
\end{aligned}
$$

A straightforward calculation shows that

$$
\begin{aligned}
& P(t) z_{\lambda}(t)=T(t, s) P(s) z_{\lambda}(s)+\int_{s}^{t} T(t, \sigma(\tau)) P(\sigma(\tau)) B(\tau, \lambda) z_{\lambda}(\tau) \Delta \tau, \\
& Q(t) z_{\lambda}(t)=T(t, s) Q(s) z_{\lambda}(s)+\int_{s}^{t} T(t, \sigma(\tau)) Q(\sigma(\tau)) B(\tau, \lambda) z_{\lambda}(\tau) \Delta \tau
\end{aligned}
$$

and

$$
z_{\lambda}(t)=P(t) z_{\lambda}(t)+Q(t) z_{\lambda}(t), \quad t \in \mathbb{T} .
$$


It follows from (3.20), (2.2) and (2.3) that

$$
Q(s) z_{\lambda}(s)=T(s, t) Q(t) z_{\lambda}(t)-\int_{s}^{t} T(s, \sigma(\tau)) Q(\sigma(\tau)) B(\tau, \lambda) z_{\lambda}(\tau) \Delta \tau
$$

and

$$
\|T(s, t) Q(t)\| \leq K L_{2}\left(t, \kappa_{1}\right) e_{\ominus b}(t, s) \leq K L_{2}\left(t, \kappa_{1}\right) e_{\ominus b}\left(t, \kappa_{1}\right) e_{\ominus b}\left(\kappa_{1}, s\right), \quad \kappa_{1} \leq t .
$$

We note that

$$
\begin{aligned}
& \int_{s}^{\infty}\left\|T(s, \sigma(\tau)) Q(\sigma(\tau)) B(\tau, \lambda) z_{\lambda}(\tau)\right\| \Delta \tau \\
& \quad \leq K c \int_{s}^{\infty} e_{\ominus b}(\sigma(\tau), s) \frac{L_{2}\left(\sigma(\tau), \kappa_{1}\right)}{L^{*}\left(\sigma(\tau), \kappa_{1}\right)} \Delta \tau \sup _{\tau \geq s}\left\|z_{\lambda}(\tau)\right\| \\
& \quad \leq K c M_{1} \sup _{\tau \geq s}\left\|z_{\lambda}(\tau)\right\|<\infty
\end{aligned}
$$

for $\kappa_{1} \leq s$ and

$$
\begin{aligned}
\int_{s}^{\infty} & \left\|T(s, \sigma(\tau)) Q(\sigma(\tau)) B(\tau, \lambda) z_{\lambda}(\tau)\right\| \Delta \tau \\
\leq & K c\left(\int_{s}^{\kappa_{2}} e_{\ominus b}(\sigma(\tau), s) \frac{L_{2}\left(\kappa_{2}, \sigma(\tau)\right)}{L^{*}\left(\kappa_{2}, \sigma(\tau)\right)} \Delta \tau\right. \\
& \left.+\int_{\kappa_{1}}^{\infty} e_{\ominus b}(\sigma(\tau), s) \frac{L_{1}\left(\sigma(\tau), \kappa_{1}\right)}{L^{*}\left(\sigma(\tau), \kappa_{1}\right)} \Delta \tau\right) \sup _{\tau \geq s}\left\|z_{\lambda}(\tau)\right\| \\
\leq & K c\left(M_{1}+M_{2}\right) \sup _{\tau \geq s}\left\|z_{\lambda}(\tau)\right\|<\infty .
\end{aligned}
$$

for $s \leq \kappa_{2}$. Then

$$
Q(s) z_{\lambda}(s)=-\int_{s}^{\infty} T(s, \sigma(\tau)) Q(\sigma(\tau)) B(\tau, \lambda) z_{\lambda}(\tau) \Delta \tau
$$

when letting $t \rightarrow \infty$ in (3.21). Consequently,

$$
\begin{aligned}
Q(t) z_{\lambda}(t)= & -\int_{s}^{\infty} T(t, \sigma(\tau)) Q(\sigma(\tau)) B(\tau, \lambda) z_{\lambda}(\tau) \Delta \tau \\
& +\int_{s}^{t} T(t, \sigma(\tau)) Q(\sigma(\tau)) B(\tau, \lambda) z_{\lambda}(\tau) \Delta \tau \\
= & -\int_{t}^{\infty} T(t, \sigma(\tau)) Q(\sigma(\tau)) B(\tau, \lambda) z_{\lambda}(\tau) \Delta \tau,
\end{aligned}
$$

which means that (3.18) holds.

For each $\lambda \in Y$, we let $z_{\lambda}(t)=\widehat{T}_{\lambda}(t, s) \widetilde{P}_{\lambda}(s) \xi, t \geq s, \xi \in X$. It is clear that $\widehat{T}_{\lambda}\left(t, \kappa_{1}\right) U_{\lambda}\left(\kappa_{1}, \kappa_{1}\right)$ and $U_{\lambda}\left(t, \kappa_{1}\right)$ are solutions of (3.1) with the same initial value $U_{\lambda}\left(\kappa_{1}, \kappa_{1}\right)$. Then

$$
\begin{aligned}
z_{\lambda}(t) & =\widehat{T}_{\lambda}(t, s) \widetilde{P}_{\lambda}(s) \xi=\widetilde{P}_{\lambda}(t) \widehat{T}_{\lambda}(t, s) \xi=\widehat{T}_{\lambda}\left(t, \kappa_{1}\right) U_{\lambda}\left(\kappa_{1}, \kappa_{1}\right) \widehat{T}_{\lambda}\left(\kappa_{1}, t\right) \widehat{T}_{\lambda}(t, s) \xi \\
& =\widehat{T}_{\lambda}\left(t, \kappa_{1}\right) U_{\lambda}\left(\kappa_{1}, \kappa_{1}\right) \widehat{T}_{\lambda}\left(\kappa_{1}, s\right) \xi=U_{\lambda}\left(t, \kappa_{1}\right) \widehat{T}_{\lambda}\left(\kappa_{1}, s\right) \xi
\end{aligned}
$$


is a bounded solution of (3.1) with the initial value $z_{\lambda}(s)=\widetilde{P}_{\lambda}(s) \xi$ since $U_{\lambda}\left(t, \kappa_{1}\right)$ is bounded for $t \in \mathbb{T}$. It follows from (3.18) that

$$
\begin{aligned}
\widehat{T}_{\lambda}(t, s) \widetilde{P}_{\lambda}(s) \xi= & T(t, s) P(s) \widetilde{P}_{\lambda}(s) \xi+\int_{s}^{t} T(t, \sigma(\tau)) P(\sigma(\tau)) B(\tau, \lambda) \widetilde{P}_{\lambda}(\tau) \widehat{T}_{\lambda}(\tau, s) \xi \Delta \tau \\
& -\int_{t}^{\infty} T(t, \sigma(\tau)) Q(\sigma(\tau)) B(\tau, \lambda) \widetilde{P}_{\lambda}(\tau) \widehat{T}_{\lambda}(\tau, s) \xi \Delta \tau, \quad t \geq s .
\end{aligned}
$$

By (2.2), (2.3), $\left(\mathrm{a}_{1}\right)$ and $\left(\mathrm{a}_{2}\right)$, we get

$$
\begin{aligned}
& \int_{s}^{t}\|T(t, \sigma(\tau)) P(\sigma(\tau))\|\|B(\tau, \lambda)\|\left\|\widetilde{P}_{\lambda}(\tau) \widehat{T}_{\lambda}(\tau, s) \xi\right\| \Delta \tau \\
& \quad \leq K c \int_{s}^{t} \frac{L_{1}\left(\sigma(\tau), \kappa_{1}\right) e_{\ominus a}(t, \sigma(\tau))}{L^{*}\left(\sigma(\tau), \kappa_{1}\right)}\left\|\widetilde{P}_{\lambda}(\tau) \widehat{T}_{\lambda}(\tau, s)\right\|\left\|\widetilde{P}_{\lambda}(s) \xi\right\| \Delta \tau \\
& \quad \leq K c \int_{s}^{t} \frac{L_{1}\left(\sigma(\tau), \kappa_{1}\right) e_{\ominus a}(t, \sigma(\tau))}{L^{*}\left(\sigma(\tau), \kappa_{1}\right)} e_{\ominus a}(\tau, s) L_{1}\left(s, \kappa_{1}\right) \Delta \tau\left\|\widetilde{P}_{\lambda} \widehat{T}_{\lambda}\right\|_{1}^{1}\left\|\widetilde{P}_{\lambda}(s) \xi\right\| \\
& \quad \leq K c e_{\ominus a}(t, s) L_{1}\left(s, \kappa_{1}\right) \int_{s}^{t}(1+a \mu(\tau)) \frac{L_{1}\left(\sigma(\tau), \kappa_{1}\right)}{L^{*}\left(\sigma(\tau), \kappa_{1}\right)} \Delta \tau\left\|\widetilde{P}_{\lambda} \widehat{T}_{\lambda}\right\|_{1}^{1}\left\|\widetilde{P}_{\lambda}(s) \xi\right\| \\
& \quad \leq K c\left(1+[a \mu]^{*}\right) M_{1} L_{1}\left(s, \kappa_{1}\right) e_{\ominus a}(t, s)\left\|\widetilde{P}_{\lambda} \widehat{T}_{\lambda}\right\|_{1}^{1}\left\|\widetilde{P}_{\lambda}(s) \xi\right\|
\end{aligned}
$$

and

$$
\begin{aligned}
& \int_{t}^{\infty}\|T(t, \sigma(\tau)) Q(\sigma(\tau))\|\|B(\tau, \lambda)\|\left\|\widetilde{P}_{\lambda}(\tau) \widehat{T}_{\lambda}(\tau, s) \xi\right\| \Delta \tau \\
& \quad \leq K c \int_{t}^{\infty} \frac{L_{2}\left(\sigma(\tau), \kappa_{1}\right) e_{\ominus b}(\sigma(\tau), t)}{L^{*}\left(\sigma(\tau), \kappa_{1}\right)}\left\|\widetilde{P}_{\lambda}(\tau) \widehat{T}_{\lambda}(\tau, s)\right\|\left\|\widetilde{P}_{\lambda}(s) \xi\right\| \Delta \tau \\
& \quad \leq K c \int_{t}^{\infty} \frac{L_{2}\left(\sigma(\tau), \kappa_{1}\right) e_{\ominus b}(\sigma(\tau), t)}{L^{*}\left(\sigma(\tau), \kappa_{1}\right)} e_{\ominus a}(\tau, s) L_{1}\left(s, \kappa_{1}\right) \Delta \tau\left\|\widetilde{P}_{\lambda} \widehat{T}_{\lambda}\right\|_{1}^{1}\left\|\widetilde{P}_{\lambda}(s) \xi\right\| \\
& \quad \leq K c e_{\ominus a}(t, s) L_{1}\left(s, \kappa_{1}\right) \int_{t}^{\infty} \frac{1}{1+b \mu(\tau)} \frac{L_{2}\left(\sigma(\tau), \kappa_{1}\right)}{L^{*}\left(\sigma(\tau), \kappa_{1}\right)} \Delta \tau\left\|\widetilde{P}_{\lambda} \widehat{T}_{\lambda}\right\|_{1}^{1}\left\|\widetilde{P}_{\lambda}(s) \xi\right\| \\
& \quad \leq K c M_{1} L_{1}\left(s, \kappa_{1}\right) e_{\ominus a}(t, s)\left\|\widetilde{P}_{\lambda} \widehat{T}_{\lambda}\right\|_{1}^{1}\left\|\widetilde{P}_{\lambda}(s) \xi\right\|
\end{aligned}
$$

for $\kappa_{1} \leq s$. Then

$$
\begin{aligned}
& \left\|\widehat{T}_{\lambda}(t, s) \widetilde{P}_{\lambda}(s) \xi\right\| \\
& \quad \leq K L_{1}\left(s, \kappa_{1}\right) e_{\ominus a}(t, s)\left\|\widetilde{P}_{\lambda}(s) \xi\right\| \\
& \quad+K c\left(2+[a \mu]^{*}\right) M_{1} L_{1}\left(s, \kappa_{1}\right) e_{\ominus a}(t, s)\left\|\widetilde{P}_{\lambda} \widehat{T}_{\lambda}\right\|_{1}^{1}\left\|\widetilde{P}_{\lambda}(s) \xi\right\|, \quad s \geq \kappa_{1} .
\end{aligned}
$$

For $s \leq \kappa_{2} \leq \kappa_{1} \leq t$, we have

$$
\begin{aligned}
& \int_{s}^{t} \| T(t, \sigma(\tau)) P(\sigma(\tau))\|\| B(\tau, \lambda)\|\| \widetilde{P}_{\lambda}(\tau) \widehat{T}_{\lambda}(\tau, s) \xi \| \Delta \tau \\
& \leq K c \int_{s}^{\kappa_{2}} \frac{L_{1}\left(\kappa_{2}, \sigma(\tau)\right) e_{\ominus a}(t, \sigma(\tau))}{L^{*}\left(\kappa_{2}, \sigma(\tau)\right)}\left\|\widetilde{P}_{\lambda}(\tau) \widehat{T}_{\lambda}(\tau, s)\right\|\left\|\widetilde{P}_{\lambda}(s) \xi\right\| \Delta \tau \\
& \quad+K c \int_{\kappa_{1}}^{t} \frac{L_{1}\left(\sigma(\tau), \kappa_{1}\right) e_{\ominus a}(t, \sigma(\tau))}{L^{*}\left(\sigma(\tau), \kappa_{1}\right)}\left\|\widetilde{P}_{\lambda}(\tau) \widehat{T}_{\lambda}(\tau, s)\right\|\left\|\widetilde{P}_{\lambda}(s) \xi\right\| \Delta \tau
\end{aligned}
$$




$$
\begin{aligned}
\leq & K c \int_{s}^{\kappa_{2}} \frac{L_{1}\left(\kappa_{2}, \sigma(\tau)\right) e_{\ominus a}(t, \sigma(\tau))}{L^{*}\left(\kappa_{2}, \sigma(\tau)\right)} e_{\ominus a}(\tau, s) L_{1}\left(\kappa_{2}, s\right) \Delta \tau\left\|\widetilde{P}_{\lambda} \widehat{T}_{\lambda}\right\|_{1}^{2}\left\|\widetilde{P}_{\lambda}(s) \xi\right\| \\
& +K c \int_{\kappa_{1}}^{t} \frac{L_{1}\left(\sigma(\tau), \kappa_{1}\right) e_{\ominus a}(t, \sigma(\tau))}{L^{*}\left(\sigma(\tau), \kappa_{1}\right)} e_{\ominus a}(\tau, s) L_{1}\left(\kappa_{2}, s\right) \Delta \tau\left\|\widetilde{P}_{\lambda} \widehat{T}_{\lambda}\right\|_{1}^{2}\left\|\widetilde{P}_{\lambda}(s) \xi\right\| \\
\leq & K c L_{1}\left(\kappa_{2}, s\right) e_{\ominus a}(t, s)\left\|\widetilde{P}_{\lambda} \widehat{T}_{\lambda}\right\|_{1}^{2}\left\|\widetilde{P}_{\lambda}(s) \xi\right\| \int_{s}^{\kappa_{2}}(1+a \mu(\tau)) \frac{L_{1}\left(\kappa_{2}, \sigma(\tau)\right)}{L^{*}\left(\kappa_{2}, \sigma(\tau)\right)} \Delta \tau \\
& +K c L_{1}\left(\kappa_{2}, s\right) e_{\ominus a}(t, s)\left\|\widetilde{P}_{\lambda} \widehat{T}_{\lambda}\right\|_{1}^{2}\left\|\widetilde{P}_{\lambda}(s) \xi\right\| \int_{\kappa_{1}}^{t}(1+a \mu(\tau)) \frac{L_{1}\left(\sigma(\tau), \kappa_{1}\right)}{L^{*}\left(\sigma(\tau), \kappa_{1}\right)} \Delta \tau \\
\leq & K c\left(1+[a \mu]^{*}\right)\left(M_{1}+M_{2}\right) L_{1}\left(\kappa_{2}, s\right) e_{\ominus a}(t, s)\left\|\widetilde{P}_{\lambda} \widehat{T}_{\lambda}\right\|_{1}^{2}\left\|\widetilde{P}_{\lambda}(s) \xi\right\|
\end{aligned}
$$

and

$$
\begin{aligned}
& \int_{t}^{\infty}\|T(t, \sigma(\tau)) Q(\sigma(\tau))\|\|B(\tau, \lambda)\|\left\|\widetilde{P}_{\lambda}(\tau) \widehat{T}_{\lambda}(\tau, s) \xi\right\| \Delta \tau \\
& \quad \leq K c \int_{t}^{\infty} \frac{L_{2}\left(\sigma(\tau), \kappa_{1}\right) e_{\ominus b}(\sigma(\tau), t)}{L^{*}\left(\sigma(\tau), \kappa_{1}\right)}\left\|\widetilde{P}_{\lambda}(\tau) \widehat{T}_{\lambda}(\tau, s)\right\|\left\|\widetilde{P}_{\lambda}(s) \xi\right\| \Delta \tau \\
& \quad \leq K c \int_{t}^{\infty} \frac{L_{2}\left(\sigma(\tau), \kappa_{1}\right) e_{\ominus b}(\sigma(\tau), t)}{L^{*}\left(\sigma(\tau), \kappa_{1}\right)} e_{\ominus a}(\tau, s) L_{1}\left(\kappa_{2}, s\right) \Delta \tau\left\|\widetilde{P}_{\lambda} \widehat{T}_{\lambda}\right\|_{1}^{2}\left\|\widetilde{P}_{\lambda}(s) \xi\right\| \\
& \quad \leq K c L_{1}\left(\kappa_{2}, s\right) e_{\ominus a}(t, s) \int_{t}^{\infty} \frac{1}{1+b \mu(\tau)} \frac{L_{2}\left(\sigma(\tau), \kappa_{1}\right)}{L^{*}\left(\sigma(\tau), \kappa_{1}\right)} \Delta \tau\left\|\widetilde{P}_{\lambda} \widehat{T}_{\lambda}\right\|_{1}^{2}\left\|\widetilde{P}_{\lambda}(s) \xi\right\| \\
& \quad \leq K c M_{1} L_{1}\left(\kappa_{2}, s\right) e_{\ominus a}(t, s)\left\|\widetilde{P}_{\lambda} \widehat{T}_{\lambda}\right\|_{1}^{2}\left\|\widetilde{P}_{\lambda}(s) \xi\right\| .
\end{aligned}
$$

For $s \leq t \leq \kappa_{2}$, we get

$$
\begin{aligned}
& \int_{s}^{t}\|T(t, \sigma(\tau)) P(\sigma(\tau))\|\|B(\tau, \lambda)\|\left\|\widetilde{P}_{\lambda}(\tau) \widehat{T}_{\lambda}(\tau, s) \xi\right\| \Delta \tau \\
& \quad \leq K c \int_{s}^{t} \frac{L_{1}\left(\kappa_{2}, \sigma(\tau)\right) e_{\ominus a}(t, \sigma(\tau))}{L^{*}\left(\kappa_{2}, \sigma(\tau)\right)}\left\|\widetilde{P}_{\lambda}(\tau) \widehat{T}_{\lambda}(\tau, s)\right\|\left\|\widetilde{P}_{\lambda}(s) \xi\right\| \Delta \tau \\
& \quad \leq K c \int_{s}^{t} \frac{L_{1}\left(\kappa_{2}, \sigma(\tau)\right) e_{\ominus a}(t, \sigma(\tau))}{L^{*}\left(\kappa_{2}, \sigma(\tau)\right)} e_{\ominus a}(\tau, s) L_{1}\left(\kappa_{2}, s\right) \Delta \tau\left\|\widetilde{P}_{\lambda} \widehat{T}_{\lambda}\right\|_{1}^{2}\left\|\widetilde{P}_{\lambda}(s) \xi\right\| \\
& \quad \leq K c\left(1+[a \mu]^{*}\right) M_{2} L_{1}\left(\kappa_{2}, s\right) e_{\ominus a}(t, s)\left\|\widetilde{P}_{\lambda} \widehat{T}_{\lambda}\right\|_{1}^{2}\left\|\widetilde{P}_{\lambda}(s) \xi\right\|
\end{aligned}
$$

and

$$
\begin{aligned}
\int_{t}^{\infty} & \|T(t, \sigma(\tau)) Q(\sigma(\tau))\|\|B(\tau, \lambda)\|\left\|\widetilde{P}_{\lambda}(\tau) \widehat{T}_{\lambda}(\tau, s) \xi\right\| \Delta \tau \\
\leq & K c \int_{t}^{\kappa_{2}} \frac{L_{2}\left(\kappa_{2}, \sigma(\tau)\right) e_{\ominus b}(\sigma(\tau), t)}{L^{*}\left(\kappa_{2}, \sigma(\tau)\right)}\left\|\widetilde{P}_{\lambda}(\tau) \widehat{T}_{\lambda}(\tau, s)\right\|\left\|\widetilde{P}_{\lambda}(s) \xi\right\| \Delta \tau \\
& +K c \int_{\kappa_{1}}^{\infty} \frac{L_{2}\left(\sigma(\tau), \kappa_{1}\right) e_{\ominus b}(\sigma(\tau), t)}{L^{*}\left(\sigma(\tau), \kappa_{1}\right)}\left\|\widetilde{P}_{\lambda}(\tau) \widehat{T}_{\lambda}(\tau, s)\right\|\left\|\widetilde{P}_{\lambda}(s) \xi\right\| \Delta \tau \\
\leq & K c L_{1}\left(\kappa_{2}, s\right) e_{\ominus a}(t, s)\left\|\widetilde{P}_{\lambda} \widehat{T}_{\lambda}\right\|_{1}^{2}\left\|\widetilde{P}_{\lambda}(s) \xi\right\| \int_{t}^{\kappa_{2}} \frac{1}{1+b \mu(\tau)} \frac{L_{2}\left(\kappa_{2}, \sigma(\tau)\right)}{L^{*}\left(\kappa_{2}, \sigma(\tau)\right)} \Delta \tau \\
& +K c L_{1}\left(\kappa_{2}, s\right) e_{\ominus a}(t, s)\left\|\widetilde{P}_{\lambda} \widehat{T}_{\lambda}\right\|_{1}^{2}\left\|\widetilde{P}_{\lambda}(s) \xi\right\| \int_{\kappa_{1}}^{\infty} \frac{1}{1+b \mu(\tau)} \frac{L_{2}\left(\sigma(\tau), \kappa_{1}\right)}{L^{*}\left(\sigma(\tau), \kappa_{1}\right)} \Delta \tau \\
\leq & K c\left(M_{1}+M_{2}\right) L_{1}\left(\kappa_{2}, s\right) e_{\ominus a}(t, s)\left\|\widetilde{P}_{\lambda} \widehat{T}_{\lambda}\right\|_{1}^{2}\left\|\widetilde{P}_{\lambda}(s) \xi\right\| .
\end{aligned}
$$


Then

$$
\begin{aligned}
\left\|\widehat{T}_{\lambda}(t, s) \widetilde{P}_{\lambda}(s) \xi\right\| \leq & K L_{1}\left(\kappa_{2}, s\right) e_{\ominus a}(t, s)\left\|\widetilde{P}_{\lambda}(s) \xi\right\| \\
& +K c\left(2+[a \mu]^{*}\right) M_{1} L_{1}\left(\kappa_{2}, s\right) e_{\ominus a}(t, s)\left\|\widetilde{P}_{\lambda} \widehat{T}_{\lambda}\right\|_{1}^{2}\left\|\widetilde{P}_{\lambda}(s) \xi\right\| \\
& +K c\left(2+[a \mu]^{*}\right) M_{2} L_{1}\left(\kappa_{2}, s\right) e_{\ominus a}(t, s)\left\|\widetilde{P}_{\lambda} \widehat{T}_{\lambda}\right\|_{1}^{2}\left\|\widetilde{P}_{\lambda}(s) \xi\right\|
\end{aligned}
$$

for $s \leq \kappa_{2}$. Combining (3.22) and (3.23) together gives (3.16). Similarly, (3.17) holds.

Lemma 3.7 We have

$$
\begin{aligned}
& \left\|\widehat{T}_{\lambda}(t, s) \widehat{P}_{\lambda}(s)\right\| \leq\left\{\begin{array}{ll}
\widehat{K} L_{1}\left(s, \kappa_{1}\right) e_{\ominus a}(t, s)\left\|\widehat{P}_{\lambda}(s)\right\|, & \kappa_{1} \leq s, \\
\widehat{K} L_{1}\left(\kappa_{2}, s\right) e_{\ominus a}(t, s)\left\|\widehat{P}_{\lambda}(s)\right\|, & s \leq \kappa_{2},
\end{array} \quad s \leq t,\right. \\
& \left\|\widehat{T}_{\lambda}(t, s) \widehat{Q}_{\lambda}(s)\right\| \leq\left\{\begin{array}{ll}
\widehat{K} L_{2}\left(s, \kappa_{1}\right) e_{\ominus b}(s, t)\left\|\widehat{Q}_{\lambda}(s)\right\|, & \kappa_{1} \leq s, \\
\widehat{K} L_{2}\left(\kappa_{2}, s\right) e_{\ominus b}(s, t)\left\|\widehat{Q}_{\lambda}(s)\right\|, & s \leq \kappa_{2},
\end{array} \quad t \leq s .\right.
\end{aligned}
$$

Proof According to Lemma 3.4, we get

$$
\begin{aligned}
& S_{\lambda}\left(\kappa_{1}\right) P\left(\kappa_{1}\right)=\left(\widetilde{P}_{\lambda}\left(\kappa_{1}\right)+\widetilde{Q}_{\lambda}\left(\kappa_{1}\right)\right) P\left(\kappa_{1}\right)=\widetilde{P}_{\lambda}\left(\kappa_{1}\right), \\
& S_{\lambda}\left(\kappa_{1}\right) Q\left(\kappa_{1}\right)=\left(\widetilde{P}_{\lambda}\left(\kappa_{1}\right)+\widetilde{Q}_{\lambda}\left(\kappa_{1}\right)\right) Q\left(\kappa_{1}\right)=\widetilde{Q}_{\lambda}\left(\kappa_{1}\right) .
\end{aligned}
$$

Let $S_{\lambda}(t)=\widehat{T}_{\lambda}\left(t, \kappa_{1}\right) S_{\lambda}\left(\kappa_{1}\right) \widehat{T}_{\lambda}\left(\kappa_{1}, t\right), t \in \mathbb{T}, \lambda \in Y$. By using (3.15), we obtain

$$
\begin{aligned}
\widehat{P}_{\lambda}(t) S_{\lambda}(t) & =\widehat{T}_{\lambda}\left(t, \kappa_{1}\right) S_{\lambda}\left(\kappa_{1}\right) P\left(\kappa_{1}\right) S_{\lambda}^{-1}\left(\kappa_{1}\right) \widehat{T}_{\lambda}\left(\kappa_{1}, t\right) \widehat{T}_{\lambda}\left(t, \kappa_{1}\right) S_{\lambda}\left(\kappa_{1}\right) \widehat{T}_{\lambda}\left(\kappa_{1}, t\right) \\
& =\widehat{T}_{\lambda}\left(t, \kappa_{1}\right) S_{\lambda}\left(\kappa_{1}\right) P\left(\kappa_{1}\right) \widehat{T}_{\lambda}\left(\kappa_{1}, t\right)=\widehat{T}_{\lambda}\left(t, \kappa_{1}\right) \widetilde{P}_{\lambda}\left(\kappa_{1}\right) \widehat{T}_{\lambda}\left(\kappa_{1}, t\right)=\widetilde{P}_{\lambda}(t) .
\end{aligned}
$$

On the other hand,

$$
\widehat{Q}_{\lambda}(t) S_{\lambda}(t)=\widehat{T}_{\lambda}\left(t, \kappa_{1}\right) S_{\lambda}\left(\kappa_{1}\right) Q\left(\kappa_{1}\right) \widehat{T}_{\lambda}\left(\kappa_{1}, t\right)=\widehat{T}_{\lambda}\left(t, \kappa_{1}\right) \widetilde{Q}_{\lambda}\left(\kappa_{1}\right) \widehat{T}_{\lambda}\left(\kappa_{1}, t\right)=\widetilde{Q}_{\lambda}(t) .
$$

Then $\operatorname{Im} \widehat{P}_{\lambda}(t)=\operatorname{Im} \widetilde{P}_{\lambda}(t)$ and $\operatorname{Im} \widehat{Q}_{\lambda}(t)=\operatorname{Im} \widetilde{Q}_{\lambda}(t)$ since $S_{\lambda}(t)$ is invertible. It follows from Lemma 3.6 that

$$
\left\|\widehat{T}_{\lambda}(t, s) \widehat{P}_{\lambda}(s)\right\| \leq\left\|\widehat{T}_{\lambda}(t, s)\left|\operatorname{Im} \widehat{P}_{\lambda}(s)\|\| \widehat{P}_{\lambda}(s)\|\leq\| \widehat{T}_{\lambda}(t, s)\right| \operatorname{Im} \widetilde{P}_{\lambda}(s)\right\|\left\|\widehat{P}_{\lambda}(s)\right\|, \quad s \leq t
$$

and

$$
\left\|\widehat{T}_{\lambda}(t, s) \widehat{Q}_{\lambda}(s)\right\| \leq\left\|\widehat{T}_{\lambda}(t, s)\left|\operatorname{Im} \widehat{Q}_{\lambda}(s)\|\| \widehat{Q}_{\lambda}(s)\|\leq\| \widehat{T}_{\lambda}(t, s)\right| \operatorname{Im} \widetilde{Q}_{\lambda}(s)\right\|\left\|\widehat{Q}_{\lambda}(s)\right\|, \quad t \leq s,
$$

which yield the desired inequalities.

Lemma 3.8 For each $\lambda \in Y$, we have

$$
\begin{aligned}
& \left\|\widehat{P}_{\lambda}(t)\right\| \leq \begin{cases}K\left(L_{1}\left(t, \kappa_{1}\right)+L_{2}\left(t, \kappa_{1}\right)\right) /\left(1-2 K \widehat{K} c\left(1+[a \mu]^{*}\right)\left(M_{1}+M_{2}\right)\right), & \kappa_{1} \leq t, \\
K\left(L_{1}\left(\kappa_{2}, t\right)+L_{2}\left(\kappa_{2}, t\right)\right) /\left(1-2 K \widehat{K} c\left(1+[a \mu]^{*}\right)\left(M_{1}+M_{2}\right)\right), & t \leq \kappa_{2},\end{cases} \\
& \left\|\widehat{Q}_{\lambda}(t)\right\| \leq \begin{cases}K\left(L_{1}\left(t, \kappa_{1}\right)+L_{2}\left(t, \kappa_{1}\right)\right) /\left(1-2 K \widehat{K} c\left(1+[a \mu]^{*}\right)\left(M_{1}+M_{2}\right)\right), & \kappa_{1} \leq t, \\
K\left(L_{1}\left(\kappa_{2}, t\right)+L_{2}\left(\kappa_{2}, t\right)\right) /\left(1-2 K \widehat{K} c\left(1+[a \mu]^{*}\right)\left(M_{1}+M_{2}\right)\right), & t \leq \kappa_{2} .\end{cases}
\end{aligned}
$$


Proof For each $\lambda \in Y$ and any $\xi \in X$, we set

$$
z_{\lambda}(t)=\widehat{T}_{\lambda}(t, s) \widehat{P}_{\lambda}(s) \xi, \quad s \leq t \quad \text { and } \quad z_{\lambda}(t)=\widehat{T}_{\lambda}(t, s) \widehat{Q}_{\lambda}(s) \xi, \quad t \leq s
$$

It follows from Lemma 3.7 that $z_{\lambda}(t)_{(s \leq t)}$ and $z_{\lambda}(t)_{(t \leq s)}$ are bounded solutions of (3.1). Combining (3.6) and (3.8) together gives

$$
\begin{aligned}
\widehat{T}_{\lambda}(t, s) \widehat{P}_{\lambda}(s) \xi= & T(t, s) P(s) \widehat{P}_{\lambda}(s) \xi+\int_{s}^{t} T(t, \sigma(\tau)) P(\sigma(\tau)) B(\tau, \lambda) \widehat{P}_{\lambda}(\tau) \widehat{T}_{\lambda}(\tau, s) \xi \Delta \tau \\
& -\int_{t}^{\infty} T(t, \sigma(\tau)) Q(\sigma(\tau)) B(\tau, \lambda) \widehat{P}_{\lambda}(\tau) \widehat{T}_{\lambda}(\tau, s) \xi \Delta \tau
\end{aligned}
$$

and

$$
\begin{aligned}
\widehat{T}_{\lambda}(t, s) \widehat{Q}_{\lambda}(s) \xi= & T(t, s) Q(s) \widehat{Q}_{\lambda}(s) \xi+\int_{-\infty}^{t} T(t, \sigma(\tau)) P(\sigma(\tau)) B(\tau, \lambda) \widehat{Q}_{\lambda}(\tau) \widehat{T}_{\lambda}(\tau, s) \xi \Delta \tau \\
& -\int_{t}^{s} T(t, \sigma(\tau)) Q(\sigma(\tau)) B(\tau, \lambda) \widehat{Q}_{\lambda}(\tau) \widehat{T}_{\lambda}(\tau, s) \xi \Delta \tau .
\end{aligned}
$$

Taking $t=s$ leads to

$$
Q(t) \widehat{P}_{\lambda}(t) \xi=-\int_{t}^{\infty} T(t, \sigma(\tau)) Q(\sigma(\tau)) B(\tau, \lambda) \widehat{P}_{\lambda}(\tau) \widehat{T}_{\lambda}(\tau, t) \xi \Delta \tau
$$

and

$$
P(t) \widehat{Q}_{\lambda}(t) \xi=\int_{-\infty}^{t} T(t, \sigma(\tau)) P(\sigma(\tau)) B(\tau, \lambda) \widehat{Q}_{\lambda}(\tau) \widehat{T}_{\lambda}(\tau, t) \xi \Delta \tau .
$$

By using Lemma 3.7 and $\left(\mathrm{a}_{2}\right)$, for $\kappa_{1} \leq t$, we have

$$
\begin{aligned}
\left\|Q(t) \widehat{P}_{\lambda}(t)\right\| & \leq K \widehat{K} c \int_{t}^{\infty} \frac{L_{2}\left(\sigma(\tau), \kappa_{1}\right) e_{\ominus b}(\sigma(\tau), t)}{L^{*}\left(\sigma(\tau), \kappa_{1}\right)} L_{1}\left(t, \kappa_{1}\right) e_{\ominus a}(\tau, t) \Delta \tau\left\|\widehat{P}_{\lambda}(t)\right\| \\
& \leq K \widehat{K} c \int_{t}^{\infty} \frac{L_{2}\left(\sigma(\tau), \kappa_{1}\right)}{L^{*}\left(\sigma(\tau), \kappa_{1}\right)} L_{1}\left(t, \kappa_{1}\right) \Delta \tau\left\|\widehat{P}_{\lambda}(t)\right\| \\
& \leq K \widehat{K} c \int_{t}^{\infty} \frac{L_{2}\left(\sigma(\tau), \kappa_{1}\right) L_{1}\left(\tau, \kappa_{1}\right)}{L^{*}\left(\sigma(\tau), \kappa_{1}\right)} \frac{L_{1}\left(t, \kappa_{1}\right)}{L_{1}\left(\tau, \kappa_{1}\right)} \Delta \tau\left\|\widehat{P}_{\lambda}(t)\right\| \\
& \leq K \widehat{K} c M_{1}\left\|\widehat{P}_{\lambda}(t)\right\|
\end{aligned}
$$

and

$$
\begin{aligned}
\left\|P(t) \widehat{Q}_{\lambda}(t)\right\| \leq & K \widehat{K} c \int_{-\infty}^{\kappa_{2}} \frac{L_{1}\left(\kappa_{2}, \sigma(\tau)\right) e_{\ominus a}(t, \sigma(\tau))}{L^{*}\left(\kappa_{2}, \sigma(\tau)\right)} L_{2}\left(t, \kappa_{1}\right) e_{\ominus b}(t, \tau) \Delta \tau\left\|\widehat{Q}_{\lambda}(t)\right\| \\
& +K \widehat{K} c \int_{\kappa_{1}}^{t} \frac{L_{1}\left(\sigma(\tau), \kappa_{1}\right) e_{\ominus a}(t, \sigma(\tau))}{L^{*}\left(\sigma(\tau), \kappa_{1}\right)} L_{2}\left(t, \kappa_{1}\right) e_{\ominus b}(t, \tau) \Delta \tau\left\|\widehat{Q}_{\lambda}(t)\right\| \\
\leq & K \widehat{K} c\left(1+[a \mu]^{*}\right)\left\|\widehat{Q}_{\lambda}(t)\right\| \\
& \times\left(\int_{-\infty}^{\kappa_{2}} \frac{L_{1}\left(\kappa_{2}, \sigma(\tau)\right) L_{2}\left(\tau, \kappa_{1}\right)}{L^{*}\left(\kappa_{2}, \sigma(\tau)\right)} \frac{e_{\ominus(a \oplus b)}\left(t, \kappa_{1}\right) L_{2}\left(t, \kappa_{1}\right)}{e_{\ominus(a \oplus b)}\left(\tau, \kappa_{1}\right) L_{2}\left(\tau, \kappa_{1}\right)} \Delta \tau\right.
\end{aligned}
$$




$$
\begin{aligned}
& \left.+\int_{\kappa_{1}}^{t} \frac{L_{1}\left(\sigma(\tau), \kappa_{1}\right) L_{2}\left(\tau, \kappa_{1}\right)}{L^{*}\left(\sigma(\tau), \kappa_{1}\right)} \frac{e_{\ominus(a \oplus b)}\left(t, \kappa_{1}\right) L_{2}\left(t, \kappa_{1}\right)}{e_{\ominus(a \oplus b)}\left(\tau, \kappa_{1}\right) L_{2}\left(\tau, \kappa_{1}\right)} \Delta \tau\right) \\
\leq & K \widehat{K} c\left\|\widehat{Q}_{\lambda}(t)\right\|\left(1+[a \mu]^{*}\right)\left(M_{1}+M_{2}\right) .
\end{aligned}
$$

For $t \leq \kappa_{2}$, we get

$$
\begin{aligned}
\left\|P(t) \widehat{Q}_{\lambda}(t)\right\| & \leq K \widehat{K} c\left\|\widehat{Q}_{\lambda}(t)\right\| \int_{-\infty}^{t} \frac{L_{1}\left(\kappa_{2}, \sigma(\tau)\right) e_{\ominus a}(t, \sigma(\tau))}{L^{*}\left(\kappa_{2}, \sigma(\tau)\right)} L_{2}\left(\kappa_{2}, t\right) e_{\ominus b}(t, \tau) \Delta \tau \\
& \leq K \widehat{K} c\left\|\widehat{Q}_{\lambda}(t)\right\| \int_{-\infty}^{t} \frac{L_{1}\left(\kappa_{2}, \sigma(\tau)\right) L_{2}\left(\kappa_{2}, \tau\right)}{L^{*}\left(\kappa_{2}, \sigma(\tau)\right)} \frac{L_{2}\left(\kappa_{2}, t\right)}{L_{2}\left(\kappa_{2}, \tau\right)} \Delta \tau \\
& \leq K \widehat{K} c\left\|\widehat{Q}_{\lambda}(t)\right\| M_{2}
\end{aligned}
$$

and

$$
\begin{aligned}
\left\|Q(t) \widehat{P}_{\lambda}(t)\right\| \leq & K \widehat{K} c\left\|\widehat{P}_{\lambda}(t)\right\| \int_{t}^{\kappa_{2}} \frac{L_{2}\left(\kappa_{2}, \sigma(\tau)\right) e_{\ominus b}(\sigma(\tau), t)}{L^{*}\left(\kappa_{2}, \sigma(\tau)\right)} L_{1}\left(\kappa_{2}, t\right) e_{\ominus a}(\tau, t) \Delta \tau \\
& +K \widehat{K} c\left\|\widehat{P}_{\lambda}(t)\right\| \int_{\kappa_{1}}^{\infty} \frac{L_{2}\left(\sigma(\tau), \kappa_{1}\right) e_{\ominus b}(\sigma(\tau), t)}{L^{*}\left(\sigma(\tau), \kappa_{1}\right)} L_{1}\left(\kappa_{2}, t\right) e_{\ominus a}(\tau, t) \Delta \tau \\
\leq & K \widehat{K} c\left\|\widehat{P}_{\lambda}(t)\right\| \int_{t}^{\kappa_{2}} \frac{L_{2}\left(\kappa_{2}, \sigma(\tau)\right) L_{1}\left(\kappa_{2}, \tau\right)}{L^{*}\left(\kappa_{2}, \sigma(\tau)\right)} \frac{e_{\ominus(a \oplus b)}\left(\kappa_{2}, t\right) L_{1}\left(\kappa_{2}, t\right)}{e_{\ominus(a \oplus b)}\left(\kappa_{2}, \tau\right) L_{1}\left(\kappa_{2}, \tau\right)} \Delta \tau \\
& +K \widehat{K} c\left\|\widehat{P}_{\lambda}(t)\right\| \int_{\kappa_{1}}^{\infty} \frac{L_{2}\left(\sigma(\tau), \kappa_{1}\right) L_{1}\left(\kappa_{2}, \tau\right)}{L^{*}\left(\sigma(\tau), \kappa_{1}\right)} \frac{e_{\ominus(a \oplus b)}\left(\kappa_{2}, t\right) L_{1}\left(\kappa_{2}, t\right)}{e_{\ominus(a \oplus b)}\left(\kappa_{2}, \tau\right) L_{1}\left(\kappa_{2}, \tau\right)} \Delta \tau \\
\leq & K \widehat{K} c\left\|\widehat{P}_{\lambda}(t)\right\|\left(M_{1}+M_{2}\right) .
\end{aligned}
$$

Then

$$
\left\|Q(t) \widehat{P}_{\lambda}(t)\right\| \leq K \widehat{K} c\left\|\widehat{P}_{\lambda}(t)\right\|\left(M_{1}+M_{2}\right)
$$

and

$$
\left\|P(t) \widehat{Q}_{\lambda}(t)\right\| \leq K \widehat{K} c\left\|\widehat{Q}_{\lambda}(t)\right\|\left(1+[a \mu]^{*}\right)\left(M_{1}+M_{2}\right) .
$$

For $\kappa_{1} \leq t$, one has

$$
\begin{aligned}
\left\|\widehat{P}_{\lambda}(t)\right\| & \leq\left\|\widehat{P}_{\lambda}(t)-P(t)\right\|+\|P(t)\|=\left\|\widehat{P}_{\lambda}(t)-P(t) \widehat{P}_{\lambda}(t)-P(t)+P(t) \widehat{P}_{\lambda}(t)\right\|+\|P(t)\| \\
& =\left\|Q(t) \widehat{P}_{\lambda}(t)-P(t) \widehat{Q}_{\lambda}(t)\right\|+\|P(t)\| \leq\left\|Q(t) \widehat{P}_{\lambda}(t)\right\|+\left\|P(t) \widehat{Q}_{\lambda}(t)\right\|+\|P(t)\| \\
& \leq K \widehat{K} c\left(1+[a \mu]^{*}\right)\left(M_{1}+M_{2}\right)\left(\left\|\widehat{P}_{\lambda}(t)\right\|+\left\|\widehat{Q}_{\lambda}(t)\right\|\right)+K L_{1}\left(t, \kappa_{1}\right)
\end{aligned}
$$

and

$$
\begin{aligned}
\left\|\widehat{Q}_{\lambda}(t)\right\| & \leq\left\|\widehat{Q}_{\lambda}(t)-Q(t)\right\|+\|Q(t)\|=\left\|\widehat{P}_{\lambda}(t)-P(t)\right\|+\|Q(t)\| \\
& \leq K \widehat{K} c\left(1+[a \mu]^{*}\right)\left(M_{1}+M_{2}\right)\left(\left\|\widehat{P}_{\lambda}(t)\right\|+\left\|\widehat{Q}_{\lambda}(t)\right\|\right)+K L_{2}\left(t, \kappa_{1}\right)
\end{aligned}
$$


since $\|P(t)\| \leq K L_{1}\left(t, \kappa_{1}\right)$ and $\|Q(t)\| \leq K L_{2}\left(t, \kappa_{1}\right)$ for $\kappa_{1} \leq t$.

$$
\begin{aligned}
\left\|\widehat{P}_{\lambda}(t)\right\|+\left\|\widehat{Q}_{\lambda}(t)\right\| \leq & 2 K \widehat{K} c\left(1+[a \mu]^{*}\right)\left(M_{1}+M_{2}\right)\left(\left\|\widehat{P}_{\lambda}(t)\right\|+\|\widehat{Q}(t)\|\right) \\
& +K\left(L_{1}\left(t, \kappa_{1}\right)+L_{2}\left(t, \kappa_{1}\right)\right) .
\end{aligned}
$$

Similarly, (3.25) holds for $t \leq \kappa_{2}$.

It follows from Lemma 3.7 and Lemma 3.8 that (3.3) and (3.4) hold. Next we show that the stable subspace $\widehat{P}_{\lambda}(X)$ and the unstable subspace $\widehat{Q}_{\lambda}(X)$ are Lipschitz continuous for the parameter $\lambda$.

Lemma 3.9 $\widehat{P}_{\lambda}$ and $\widehat{Q}_{\lambda}$ are Lipschitz continuous for the parameter $\lambda$.

Proof By using Lemma 3.1 and Lemma 3.2, $U_{\lambda}$ and $V_{\lambda}$ are Lipschitz continuous for the parameter $\lambda$. It follows from (3.10) that $\widetilde{P}_{\lambda}(t)$ and $\widetilde{Q}_{\lambda}(t)$ are Lipschitz continuous since $\widehat{T}_{\lambda}$ is Lipschitz continuous for the parameter $\lambda$. If $Y$ is a finite-dimensional space, then $S_{\lambda}\left(\kappa_{1}\right)$ and $S_{\lambda}^{-1}\left(\kappa_{1}\right)$ are both Lipschitz continuous for the parameter $\lambda$. Equation (3.15) implies that the conclusion of lemma holds.

\section{Competing interests}

The authors declare that they have no competing interests.

\section{Authors' contributions}

Each author's contribution in the paper is equal, and all authors read and approved the final manuscript.

\section{Acknowledgements}

The authors are grateful to the anonymous referee for carefully reading the manuscript and for important suggestions and comments. This research is supported by the National Natural Science Foundation of China (No. 11201128) and (No. 11126269) and the youth scientific funds of Heilongjiang University (QL201007).

Received: 17 July 2013 Accepted: 28 October 2013 Published: 22 Nov 2013

\section{References}

1. Coppel, WA: Dichotomies in Stability Theory. Lecture Notes in Mathematics, vol. 629. Springer, Berlin (1978)

2. Perron, O: Die Stabilitätsfrage bei Differentialgleichungen. Math. Z. 32, 703-728 (1930)

3. Barreira, L, Fan, M, Valls, C, Zhang, JM: Parameter dependence of stable manifolds for delay equations with polynomial dichotomies. J. Dyn. Differ. Equ. 24, 101-118 (2012)

4. Barreira, L, Fan, M, Valls, C, Zhang, JM: Stable manifolds for delay equations and parameter dependence. Nonlinear Anal. TMA 75, 5824-5835 (2012)

5. Barreira, L, Valls, C: Stability of Nonautonomous Differential Equations. Lecture Notes in Math., vol. 1926. Springer, Berlin (2008)

6. Barreira, L, Valls, C: Robustness of general dichotomies. J. Funct. Anal. 257, 464-484 (2009)

7. Barreira, L, Valls, C: Polynomial growth rates. Nonlinear Anal. TMA 71, 5208-5219 (2009)

8. Bento, A, Silva, C: Nonuniform $(\mu, v)$-dichotomies and local dynamics of difference equations. Nonlinear Anal. TMA 75, 78-90 (2012)

9. Chang, XY, Zhang, JM, Qin, JH: Robustness of nonuniform $(\mu, v)$-dichotomies in Banach spaces. J. Math. Anal. Appl. 387, 582-594 (2012)

10. Jiang, LP: Generalized exponential dichotomy and global linearization. J. Math. Anal. Appl. 315, 474-490 (2006)

11. Jiang, LP: Strongly topological linearization with generalized exponential dichotomy. Nonlinear Anal. TMA 67, 1102-1110 (2007)

12. Megan, M, Sasu, B, Sasu, A: On nonuniform exponential dichotomy of evolution operators in Banach spaces. Integral Equ. Oper. Theory 44, 71-78 (2002)

13. Minda, A, Megan, M: On ( $h, k)$-stability of evolution operators in Banach spaces. Appl. Math. Lett. 24, 44-48 (2011)

14. Naulin, R, Pinto, M: Roughness of ( $h, k)$-dichotomies. J. Differ. Equ. 118, 20-35 (1995)

15. Sasu, AL, Babuția, MG, Sasu, B: Admissibility and nonuniform exponential dichotomy on the half-line. Bull. Sci. Math. 137, 466-484 (2013)

16. Barreira, L, Valls, C: Robust nonuniform dichotomies and parameter dependence. J. Math. Anal. Appl. 373, 690-708 (2011)

17. Chow, SN, Leiva, H: Existence and roughness of the exponential dichotomy for skew-product semiflows in Banach spaces. J. Differ. Equ. 120, 429-477 (1995) 
18. Naulin, R, Pinto, M: Admissible perturbations of exponential dichotomy roughness. Nonlinear Anal. TMA 31, 559-571 (1998)

19. Popescu, L: Exponential dichotomy roughness on Banach spaces. J. Math. Anal. Appl. 314, 436-454 (2006)

20. Popescu, L: Exponential dichotomy roughness and structural stability for evolution families without bounded growth and decay. Nonlinear Anal. TMA 71, 935-947 (2009)

21. Barreira, L, Fan, M, Valls, C, Zhang, JM: Robustness of nonuniform polynomial dichotomies for difference equations. Topol. Methods Nonlinear Anal. 37, 357-376 (2011)

22. Chu, J: Robustness of nonuniform behavior for discrete dynamics. Bull. Sci. Math. (2013, in press)

23. Naulin, R, Pinto, M: Stability of discrete dichotomies for linear difference systems. J. Differ. Equ. Appl. 3, 101-123 (1997)

24. Bohner, M, Peterson, A: Dynamic Equations on Time Scales: An Introduction with Applications. Birkhäuser, Boston (2001)

25. Hilger, S: Analysis on measure chains-a unified approach to continuous and discrete calculus. Results Math. 18, 18-56 (1990)

26. Agarwal, RP, Bohner, M, O'Regan, D, Peterson, A: Dynamic equations on time scales: a survey. J. Comput. Appl. Math. $141,1-26(2002)$

27. Agarwal, RP, Bohner, M: Basic calculus on time scales and some of its applications. Results Math. 35, 3-22 (1998)

28. Bohner, M, Lutz, DA: Asymptotic behavior of dynamic equations on time scales. J. Differ. Equ. Appl. 7, 21-50 (2001)

29. Hamza, AE, Oraby, KM: Stability of abstract dynamic equations on time scales. Adv. Differ. Equ. 143, 1-15 (2012)

30. Li, YK, Wang, C: Pseudo almost periodic functions and pseudo almost periodic solutions to dynamic equations on time scales. Adv. Differ. Equ. 77, 1-24 (2012)

31. Pötzsche, C: Exponential dichotomies for dynamic equations on measure chains. Nonlinear Anal. TMA 47, $873-884$ (2001)

32. Pötzsche, C: Exponential dichotomies of linear dynamic equations on measure chains under slowly varying coefficients. J. Math. Anal. Appl. 289, 317-335 (2004)

33. Siegmund, S: A spectral notion for dynamic equations on time scales. J. Comput. Appl. Math. 141, 255-265 (2002)

34. Xia, Y, Cao, J, Han, M: A new analytical method for the linearization of dynamic equations on measure chains. J. Differ. Equ. 235, 527-543 (2007)

35. Xia, YH, Li, JB, Wong, PJY: On the topological classification of dynamic equations on time scales. Nonlinear Anal., Real World Appl. 14, 2231-2248 (2013)

36. Zhang, JM, Fan, M, Zhu, HP: Existence and roughness of exponential dichotomies of linear dynamic equations on time scales. Comput. Math. Appl. 59, 2658-2675 (2010)

37. Zhang, JM, Fan, M, Zhu, HP: Necessary and sufficient criteria for the existence of exponential dichotomy on time scales. Comput. Math. Appl. 60, 2387-2398 (2010)

38. Zhang, JM, Fan, $M$, Chang, $X Y$ : Nonlinear perturbations of nonuniform exponential dichotomy on measure chains. Nonlinear Anal. TMA 75, 670-683 (2012)

10.1186/1687-1847-2013-339

Cite this article as: Zhang et al.: General exponential dichotomies on time scales and parameter dependence of roughness. Advances in Difference Equations 2013, 2013:339

\section{Submit your manuscript to a SpringerOpen ${ }^{\circ}$ journal and benefit from:}

- Convenient online submission

Rigorous peer review

- Immediate publication on acceptance

- Open access: articles freely available online

- High visibility within the field

- Retaining the copyright to your article 